\title{
A Review of the Tip-of-the-Tongue Experience
}

\author{
Alan S. Brown \\ Southern Methodist University
}

\begin{abstract}
The tip-of-the-tongue experience (TOT) has intrigued psychologists for nearly a century. R. Brown and McNeill (1966) provided the first systematic exploration of the phenomenon, and the findings since their seminal study suggest that TOTs (a) are a nearly universal experience, (b) occur about once a week, (c) increase with age, (d) are frequently elicited by proper names, (e) often enable access to the target word's first letter, (f) are often accompanied by words related to the target, and (g) are resolved during the experience about half of the time. Important questions remain concerning TOTs: (a) Are emotional reactions necessary, (b) do only low frequency targets elicit TOTs, (c) do TOTs reflect incomplete target word activation or interference from related words, and (d) do spontaneous retrievals really occur? A more precise definition of the TOT experience is needed, as well as greater uniformity in the information gathered during TOTs.
\end{abstract}

The retrieval of information from memory is a relatively automatic procedure that we take for granted because it usually is quick and effortless. On occasion, however, memory falters: We are sure that the information is in memory but are temporarily unable to access it. The tip-of-the-tongue experience (TOT) is a common label for this difficulty. This phenomenon has fascinated psychologists for a century (James, 1890). Because word retrieval is usually so rapid, examining it in a temporary "holding pattern" imposed by the TOT has the potential to reveal subtleties of normal retrieval functions, similar to how slowmotion photography clarifies the dimensions of a hummingbird's flight. However, this phenomenon has proven difficult to bring under close experimental scrutiny. In fact, it was not until three quarters of a century after James's description that the first systematic study was undertaken (R. Brown \& McNeill, 1966).

The TOT is inherently interesting to most lay persons and memory researchers. Questionnaire studies suggest that nearly everyone experiences TOTs with some regularity (see Reason, 1984). The TOT experience is mentioned in most textbooks on introductory psychology, cognition, and memory. An informal survey of 35 memory-cognition textbooks published since 1970 revealed that nearly two thirds (23) covered the TOT phenomenon. Despite numerous investigations since $R$. Brown and McNeill (1966), most textbooks focus on their study.

A structured investigation of any naturally occurring phenomenon presents unique challenges. Although R. Brown and McNeill (1966) provided an initial framework, the literature contains an eclectic assortment of techniques to produce and observe TOTs. There is inconsistency from study to study on what types of information are (a) requested from subjects dur-

I express my appreciation to Douglas Hintzman and Hildy Halliday for their helpful comments on the manuscript of this article.

Correspondence concerning this article should be addressed to Alan S. Brown, Department of Psychology, Southern Methodist University, Dallas, Texas 75275 . ing TOTs and (b) summarized in the data reports. For example, knowing how many subjects experienced TOTs and how many stimuli evoked TOTs would be useful, but most studies do not include such information. The number of recall failures would help in gauging TOT frequency, but this is rarely noted in the empirical reports.

TOTs are sometimes examined as a "second thought." As an example, Meyer and Hilterbrand (1984) investigated retrieval of information from a small semantic category-the Seven Dwarfs. "Our review of the incorrect guesses of the dwarfs' names suggests that many of the subjects were in a 'tip-of-thetongue' state" (p. 49). With this ex post facto approach, details of TOTs are indirectly inferred from other responses rather than directly provided by subjects. Such research is not included in this review.

This review is divided into nine sections. The first describes the TOT "state" and the "feeling-of-knowing" experience and offers experimental definitions; the second describes techniques to elicit and record TOT experiences; the third summarizes TOT frequency in both natural and laboratory settings. The fourth section covers types of target word information available to subjects in a TOT. The fifth and sixth sections address the likelihood of resolving TOTs within immediate (minutes) and delayed (hours to days) time frames. The seventh section presents theories on the causes of TOTs, and the eighth section summarizes individual differences related to TOTs, with respect to children, older adults, and aphasic patients. The last part of the review covers phenomena related to the TOT experience.

Traditional statistical techniques are difficult to apply in this area of research. Because some subjects fail to experience TOTs -and because some target words fail to elicit them - the application of standard statistical tests, either across subjects or across items, is problematic. This difficulty was referred to as the "fragmentary data problem" by R. Brown and McNeill (1966). Although a problem within individual investigations, consistencies across studies help establish the reliability of the findings and lessen a dependence on individual statistical tests. 


\section{Defining the TOT}

\section{Is There a TOT "State"?}

The TOT experience is often described as a TOT state, as if separate from normal waking consciousness. This is alluded to in James's (1893) original and oft-cited description of the TOT experience.

The state of our consciousness is peculiar. There is a gap therein; but no mere gap. It is a gap that is intensely active. A sort of wraith of the name is in it, beckoning us in a given direction, making us at moments tingle with the sense of our closeness and then letting us sink back without the longed-for term. If wrong names are proposed to us, this singularly definite gap acts immediately so as to negate them. They do not fit into its mould. And the gap of one word does not feel like the gap of another, all empty of content as both might seem necessarily to be when described as gaps. (p. 251)

This colorful description was supplemented by R. Brown and McNeill (1966), who noted that the signs of a TOT were distinct: "[A subject] 'seized' by a TOT state . . . would appear to be in mild torment, something like the brink of a sneeze, and if he found the word his relief was considerable" (p. 326).

Such descriptions are congruent with one's personal introspections of inner turmoil when grappling for an elusive word. However, this emotional arousal may simply reflect a reaction to the momentary retrieval failure rather than being an integral component of the TOT. Some researchers interpret an emotional reaction as a necessary part of the TOT. Gruneberg, Smith, and Winfrow (1973) did not classify a response blockage as a TOT unless subjects exhibited "clear cut emotional reactions" (p.196). Yarmey (1973) also viewed the emotional component as an essential part of a TOT. In this review, a TOT is not viewed as a separate state, and an emotional reaction is not considered a defining element. The phrase TOT state is not used, in order to avoid this implication.

\section{TOT and Feeling of Knowing}

A number of metamemory investigations have examined the feeling of knowing, or FOK, experience. Following the original series of studies by Hart $(1965,1966,1967)$, this research has consistently demonstrated that individuals can predict the likelihood of later recognizing target words presently unrecallable (see Nelson, 1984, for a review). Although related to the TOT experience, the FOK phenomenon differs in two central ways. First, TOTs occur involuntarily, whereas FOK responses are requested by the experimenter and can be made on any nonrecalled item. A second difference is that with a TOT, subjects are confident that they can eventually recall the target information, with or without additional cues. An FOK, on the other hand, assesses recognition likelihood for the missing word.

Yaniv and Meyer (1987) directly compared these two experiences by having subjects give both TOT and FOK assessments on all nonrecalled items. After retrieval failure, subjects designated whether they were experiencing a TOT (yes or no) followed by a FOK evaluation (on a 1 to 5 scale). In some instances, subjects responded no to TOT yet gave a high FOK rating, prompting Yaniv and Meyer to speculate that different introspective processes underlie these two responses.

\section{Experimental Definitions}

Most persons intuitively appreciate what a TOT is, but it is still important to define the experience for subjects. R. Brown and McNeill (1966) told subjects, "If you are unable to think of the word but feel sure that you know it and that it is on the verge of coming back to you then you are in a TOT state" (p. 327). Another typical instruction is from Koriat and Lieblich (1974): "We are concerned with that state of mind in which a person is unable to think of a word that he is certain he knows, the state of mind in which a word seems to be on the tip of one's tongue" (p. 648). Most TOT studies duplicate R. Brown and McNeill's definition, and those that do not usually stipulate that (a) the word is known but presently unavailable and (b) recall seems imminent.

This review includes only studies in which subjects were instructed about TOTs and how to report them or in which reports of subjects' momentary recall blockages clearly suggest a TOT experience. Investigations were excluded that defined TOTs in a post hoc manner, that were based on fragmentary or incomplete recall (Meyer \& Hilterbrand, 1984), or that were based on the superficial resemblance between pathological retrieval difficulties and normal subjects' TOTs (Matison, Mayeux, Rosen, \& Fahn, 1982).

\section{Eliciting and Measuring TOTs}

Because TOTs occur sporadically, a primary challenge in TOT research is evoking them. No technique has been developed to elicit a TOT with a high degree of certainty. One approach is to record TOTs as they spontaneously occur during daily activities; another involves evoking TOTs in a controlled laboratory setting.

In naturalistic investigations of TOTs, subjects either carry a diary and document TOTs as they occur (Burke, MacKay, Worthley, \& Wade, 1991; Burke, Worthley, \& Martin, 1988; Cohen \& Faulkner, 1986; Reason \& Lucas, 1984) or record TOTs at the end of each day (Sunderland, Watts, Baddeley, \& Harris, 1986). These studies usually involve an extended time period, ranging between 1 week (Sunderland et al., 1986) and 1 month (Burke et al., 1988, 1991; Cohen \& Faulkner, 1986; Reason \& Lucas, 1984), although one investigation sampled only one TOT experience per subject (Cohen \& Faulkner, 1986). Because of the effort required, such investigations involve a select group of highly motivated individuals who may not be representative of the population as a whole.

With most diary investigations, subjects are asked to provide structural features of the missing target word (number of syllables or first letter) as well as words related to the target. These studies also include questions about how the memory block was resolved, as well as the class of words (person's names, object nouns, verbs, etc.) that lead to the TOT. A limitation of diary research is that the accuracy of peripheral information about the target cannot be verified unless the target word is retrieved. Some researchers avoid this difficulty by reporting only resolved TOTs (Reason \& Lucas, 1984). Reason and Lucas suggested that the number of TOTs, as well as information available during the TOT experience, will probably be underreported with a naturalistic procedure. Although laboratory stud- 
ies are inefficient because of the low probability of TOTs, the experimenter can exercise direct control over the types of information gathered from the subjects.

In the lab, most researchers depend on "prospecting" ( $R$. Brown \& McNeill, 1966), in which the experimenter reads definitions of rare words and subjects either (a) write the target word if they know it, (b) write nothing if they do not, or (c) indicate that they are in a TOT. When in a TOT, subjects record additional information about the target word on a questionnaire form. When conducted in a group setting, the experimenter usually pauses until TOT subjects have finished filling out the questionnaire.

The information requested concerning the target word generally follows R. Brown and McNeill's (1966) model: (a) number of syllables (1 to 5), (b) initial letter, (c) words of similar sound, and (d) words of similar meaning. Subjects in R. Brown and McNeill's study were lead to expect related words to occur: "When you are in a TOT state, words that are related to the target word do almost always come to mind" (R. Brown \& McNeill, 1966, p. 327). This instruction bias pervades subsequent studies. Targets recalled before the experimenter reads them are written on the questionnaire; for targets not retrieved, subjects indicate whether the word read by the experimenter is the one they were thinking of.

Gruneberg et al. (1973) had subjects generate their own TOTs by sorting through their memories one category at a time. For subjects having difficulty generating TOTs, the experimenter supplied additional categories to explore. Although initially skeptical about such a task-trying to recall words they could not recall-subjects were successful in generating blocks once they tried. Another procedural variation had subjects talk about anything that came to mind during the TOT (Kohn et al., 1987). Kohn et al. reasoned that this was a more natural approach because TOTs appear to represent a breakdown in spoken rather than written language. In these oral efforts to resolve TOTs, many nonword fragments or phonological units came to mind that are difficult to express in a written format.

Freedman and Landauer's (1966) approach, introduced concurrently with R. Brown and McNeill's (1966), did not provide a prototype for later research, probably because it was less precise than Brown and McNeill's. When subjects could not answer a general information item, they rated how confident they were that they knew the answer. Items rated definitely know it were assumed to represent TOTs. Unfortunately, subjects were not required to provide any information concerning the TOT, making the study of limited usefulness in this review.

TOT research has been confined almost exclusively to natural category information, but list-learning tasks with artificial materials have also elicited TOTs (Ryan, Petty, \& Wenzlaff, 1982; Wearing, 1970). Using a paired-associate list, Ryan et al. investigated TOTs at the time of test by presenting the stimulus word alone. When subjects were sure they knew the response but were unable to produce it at the moment, they were said to be having a TOT. Nearly all of the subjects $(90 \%)$ claimed that the paired-associate TOTs were similar to those with natural materials. This suggests that the use of artificial materials to elicit TOTs should be pursued more seriously.

\section{TOTs and Stress}

Some anecdotal evidence suggests that TOTs are more likely when persons are under stress. Cohen and Faulkner (1986) noted that "several subjects reported that the incidence and severity of name blocks increased when they were tired, stressed, or unwell" (p. 189). Such comments were from older subjects, and Cohen and Faulkner suggested that anxiety about impending memory failure may precipitate TOTs in older adults. Mitchell (1983) found that the subject exhibiting the highest number of TOTs had just attended his aunt's funeral. He blocked on $6 \%$ of the names of pictured objects, whereas the average for this group was $1 \%$ blocks.

In a direct assessment of this relationship, Burke et al. (1991) had their subjects rate their current worry, excitement, fatigue, and sickness (less than usual [1] to more than usual [7]) at the time of each natural TOT. Fatigue was the highest of the four scales but did not vary much from the scalar midpoint of 4 for young (4.11), midage (4.76), or older (3.24) subjects. Therefore, this outcome does not support a strong relationship between arousal and naturally occurring memory blocks.

Reasoning that the stress usually experienced in association with academic examinations may precipitate TOTs, I surveyed 79 undergraduate psychology majors in advanced level courses concerning TOTs during tests. All subjects claimed to experience such TOTs at least once a semester, and $41 \%$ said they experienced them one or more times during each test. Most (75\%) acknowledged that blocks occurred more often when they were "too nervous." Investigating the incidence and resolution of TOTs during examinations could prove fruitful, especially because many students claim that test anxiety elicits memory blocks for supposedly well-learned information. It may be useful to routinely evaluate the momentary anxiety level of subjects participating in laboratory investigations, as well as the stress level preceding TOTs in diary studies.

\section{TOT Incidence}

The frequency of TOTs has been assessed in three different ways. First, subjects may provide estimates of how often they think they experience TOTs in daily living. A second technique is to have subjects record, in diary form, the TOTs that they actually experience. Finally, the incidence of TOTs is measured in laboratory retrieval tasks as a function of items rather than time.

\section{Self-Report Estimates}

When Reason (1984) asked students to assess how often they experienced various memory difficulties, the majority (51\%) claimed to have TOTs at least weekly, whereas $14 \%$ had TOTs daily. Reason's sample included undergraduate students who are likely to be frequently involved in demanding retrieval tasks, so his estimates may be high in relation to the general population. Using a sample of older adults, Sunderland et al. (1986) found that the median estimate of TOT frequency was "about once a week," which corresponds to Reason's outcome. Comparing estimates of TOT frequencies across three age groups, Burke et al. (1991) discovered weekly means of 0.76 , 
0.80 , and 0.81 for younger, midage, and older-age groups, respectively. Across these studies, it appears reasonable to conclude that self-estimates of TOT frequency in adults is approximately once a week.

\section{Incidence Over Time}

Another technique for estimating TOT frequency is through subjects' records of their naturally occurring TOTs. For instance, Sunderland et al. (1986) had participants fill out a behavioral checklist on memory difficulties every evening for 7 days, and this yielded a median of 2 TOTs per week. Reason and Lucas (1984) asked volunteers to record naturally occurring TOTs over a 4-week period and discovered an average of 2.3 TOTs, or about half of a TOT per person per week. They found similar results with a second sample: an average of 2.4 TOTs per diarist over a 4-week period. This study probably underestimates the actual TOT frequency for two reasons: (a) Each subject was given five TOT forms and told that this was all they needed to document, and (b) only resolved TOTs were considered, for which the target word was eventually retrieved.

Two other diary investigations support this speculation that Reason and Lucas's estimate of naturally occurring TOTs is low. Burke et al. (1991) (also see Burke et al., 1988) found that the mean frequency of TOTs over a 4-week period was 3.9 for younger, 5.4 for midage, and 6.6 for older adults, and Cohen and Faulkner (1986) yielded even higher monthly averages of 8.2 for younger, 7.9 for midage, and 16.1 for older adults. This substantial difference cannot be accounted for by Reason and Lucas (1984) only counting resolved TOTs. Excluding unresolved TOTs from Burke et al.'s (1991) data reduced the mean TOTs only slightly to 3.6 for younger, 5.1 for midage, and 6.4 for older adults. A more likely explanation for the lower TOT frequency in Reason and Lucas is their artificial ceiling of 5 TOTs because the range of TOTs in Burke et al. (1991) exceeded 5 in each group (younger, 1 to 7 ; midage, 1 to 17 ; older, 1 to 17 ).

These studies suggest that the self-reported naturalistic incidence of TOTs is one to two per week for younger subjects and two to four each week for older adults. This is probably an underestimate of the actual number of TOTs experienced. In the flow of everyday living, those TOTs that are resolved quickly are likely to be forgotten, as are TOTs that concern trivial information. Also, TOTs that occur when subjects are busy or preoccupied may be difficult to remember later. It would be helpful to know whether subjects become more attuned to remembering and recording TOTs by noting whether the reported frequency increases across the diary time span.

\section{Incidence Over Items}

A third way of assessing TOT incidence is in the laboratory, by what percentage of retrieval efforts result in TOTs. R. Brown and McNeill's (1966) investigation yielded TOTs on 13\% of retrieval attempts, and these were distributed across most definitions ( 48 of 49 ) and subjects ( 47 of 56 ). For $11 \%$ of all TOTs, the target word recalled was different from that designated by the experimenter, and on $35 \%$ of the TOTs the target word was not recalled and judged to differ from the designated one. $R$.
Brown and McNeill referred to the last set as negative TOTs. (They gave a brief report of a separate pilot investigation that also yielded a $13 \%$ TOT rate.) Using definitions like R. Brown and McNeill's, other studies have yielded TOT rates similar to theirs. For instance, Koriat and Lieblich $(1974,1975,1977)$ discovered that TOTs occurred on $11 \%$ of retrievals, whereas Yaniv and Meyer (1987) noted TOT rates of $18 \%$ and $15 \%$ in two separate experiments. Kozlowski (1977) found TOTs on $15 \%$ and $13 \%$ of retrievals in two studies. In a study testing only older adults, Finley and Sharp (1989) used trivia questions to elicit target words and discovered a relatively high rate of $26 \%$ TOTs.

In the Kozlowski (1977) investigation, negative TOTs were excluded so their percentages may underestimate the actual incidence of TOTs. The issue of whether to include the negative TOTs varies across investigations. Unfortunately, the distinction between positive and negative TOTs is not even reported in some studies. The ambiguity concerning negative TOTs reflects some of the definitional confusion in this area of investigation: Can a subject have a legitimate TOT on a target word not designated by the experimenter? Although impossible to score on partial recall of target-word attributes, it seems reasonable to include these negative TOTs to gain an accurate indication of the overall TOT incidence. Information about the target word provided by subjects in negative TOTs may even establish a chance baseline against which to compare the accuracy of target-word information in positive TOTs (Kohn et al., 1987).

Gardiner, Craik, and Bleasdale (1973) discovered that $15 \%$ of the retrieval efforts resulted in TOTs, but this figure may be inflated because they combined items classified in "strong feeling-of-knowing the word" and "a TOT state" to yield their TOT category. Gardiner et al. also reported a pilot investigation in which subjects simply stated when they were having a TOT. The reported incidence of $8 \%$ is probably low because it only represents TOTs resolved within $90 \mathrm{~s}$.

Rubin (1975) used the four words from R. Brown and McNeill (1966) that elicited the highest number of TOTs. Interestingly, Rubin found a TOT rate of $10 \%$, comparable to what Brown and McNeill found for their entire sample of words. This may suggest that within a certain range of difficulty, TOT incidence is related more to the number of retrieval efforts than to the specific type of information queried.

Cueing famous names with pictures, Yarmey (1973) found TOTs occurred on $14 \%$ of retrieval attempts. Similarly, Reason and Mycielska (1982) used pictures to cue names of cinema stars but restricted their subject sample to 5 persons who studied film celebrities as a hobby. Their data indicate that $7 \%$ of faces recognized by subjects yielded TOTs (estimated from their Figure 6.1). When instructed to rummage through their own memory categories, Gruneberg et al. (1973) found that all of their subjects experienced TOTs, with a range of 2 to 18 TOTs per subject. The first block occurred between 15 and 250 s after subjects began their self-directed searches, with a mean onset time of $112 \mathrm{~s}$.

Using an artificial learning task consisting of consonantvowel-consonant (CVC) pairs, Wearing (1970) had subjects recall responses to stimuli a day after original learning. For responses they could not remember, subjects rated their confidence in eventual recall on a 5-point scale with the endpoint 
defined as a TOT. Their 3\% rate of TOTs is below that found with naturalistic materials, and it is difficult to tell whether this is due to the episodic task, the nonsense syllable material, or the degree of initial learning.

Jones (1989; Jones \& Langford, 1987) has conducted experiments on the TOT experience in which he accompanied each cue definition with a prime word resembling the target in sound or meaning (or both, or neither). His purpose was to influence the number of TOTs produced, so the results do not yield an unbiased estimate of TOT frequency. Phonologically similar prime words led to TOTs on $22 \%$ of definitions, whereas phonologically unrelated words elicited TOTs $18 \%$ of the time (Jones \& Langford, 1987). The latter percentage is a less biased estimate of TOTs because the phonologically related words apparently increased TOT frequency. In a follow-up study, Jones (1989) reported separate TOT incidences for each condition: similar meaning and sound, $12 \%$; similar sound only, $13 \%$; similar meaning only, $8 \%$; and no relationship, $7 \%$. The best reflection of the natural incidence of TOTs is $7 \%$, for which target and prime words are unrelated.

In general, the rate of TOTs is consistent across investigations. With natural stimulus materials, TOT probabilities range about 5\% in either direction of R. Brown and McNeill's (1966) original $13 \%$. When such data are reported, the vast majority of both stimuli and subjects yield TOTs. The consistency in the percentage of TOTs across studies varying in the type of material and target word difficulty is puzzling. It may reflect subjects' acquiescence to the experimenter's suggestion that TOTs will be occasionally experienced, and such demand characteristics may be more pronounced when subjects are tested in groups and witness others experiencing TOTs. Would instructions without explicit reference to TOTs influence TOT probability? This consistency may also indicate that across the course of repeated retrievals of low-frequency information, memory search efforts falter a certain proportion of the time. It also may be the case that studies with a low incidence of TOTs (i.e., $1 \%$ to $2 \%$ ) are abandoned and never get published.

As a methodological note, the TOT rates in this review are percentages of total retrieval attempts. Expressing TOTs as a percentage of unsuccessful retrievals might adjust for differences in item difficulty across studies, but such information was rarely provided in the articles reviewed. It would also be useful to know some descriptive statistics on the difficulty level of the items to evaluate to what extent the differences across studies are due to the nature of the item pool.

\section{Dimensions of the Target Word}

While in a TOT, subjects often experience what appear to be fragments of the target word, or words related to the target. As James (1890) noted,

the rhythm of the lost word may be there without the sound to clothe it; or the evanescent sense of something which is the initial vowel or consonant may mock us fitfully, without growing more distinct. (p. 251)

Availability of fragmentary information suggests that the target word is activated, though not directly accessible. This may reflect the fact that words are stored as a bundle of attributes and that retrieval of one or more attributes is possible without recalling the target. In this section of the review, the categories of target-word information available during the TOT are discussed, followed by an overview of the types of target words likely to be blocked. Two issues thread their way throughout this topic. One involves whether target-word information comes to mind spontaneously or is accessed to meet the experimenter's expectations. A second issue is whether target word information reflects an access to target-word information or is merely a result of educated guessing.

\section{Similar Words}

R. Brown and McNeill (1966) instructed subjects that "when you are in a TOT state, words that are related to the target word do almost always come to mind" (p. 327). It is perhaps not surprising that subjects reported many words related to the target during TOTs. Whether these are a forced or natural aspect of TOTs, they provide useful insights into the type of target-word information available prior to retrieval.

After generating related words, $R$. Brown and McNeill's subjects classified their relationship to the target word as similar in sound (SS) or similar in meaning (SM). The majority (70\%) were SS words, but this may have been due to the emphasis on structural information in the questionnaire. Other investigations have reported the frequency of related words during TOTs (Cohen \& Faulkner, 1986; Reason \& Lucas, 1984; Yarmey, 1973) but have not provided separate counts of SS and SM words. Some investigators disregard SM words and ask subjects to report only SS words (Koriat \& Lieblich, 1974), although others do not differentiate between SS and SM words because the distinction is often ambiguous. For example, with the target word chastity, is charity an SM or SS word (Burke et al., 1991)? Kohn et al. (1987) showed that related words could be divided into $20 \%$ as SS, $54 \%$ as SM, and $25 \%$ that were related in both sound and meaning (SS-SM hybrids). They also discovered that $57 \%$ of extraneous words generated by subjects when guessing (not in a TOT) were semantically related to the target word, which probably represents the subject's ability to extrapolate from the definition provided. In summary, the SM-SS distinction may be somewhat artificial and should be interpreted cautiously.

Another dimension of related words is how often they come to mind during TOTs. R. Brown and McNeill (1966) noted the total number reported but not how many TOTs were accompanied by such words (some TOTs yielded more than one related word). Subsequent investigations, however, have found that approximately half of TOTs involve related words: 53\% (Reason \& Lucas, 1984), 50\% (Cohen \& Faulkner, 1986), and 56\% (Burke et al., 1991).

In a creative exploration into the role of related words in the TOT experience, Kozlowski (1977) used a poetic analogy. He assumed that the rhythm, rhyme, structure, and meaning of standard poetic verse leads the reader toward a target word in the same manner that an individual uses fragmentary meaning and structural information during a TOT to help locate the missing target word. Kozlowski reasoned that poets should be better able to use partial target information during a TOT because they are accustomed to using such cues in decoding po- 
etry. He played a distorted auditory version of the target word while subjects were in a TOT. This auditory stimulus preserved the target word's stress pattern, rhythm, number of syllables, some tonal information, and "sounded as if a man was speaking through a pillow" (p. 478). The poets expressed a greater awareness of the helpfulness of the auditory cue, although there was no difference between poets and nonpoets in the likelihood of resolving TOTs after hearing this cue. The poets were more introspective than nonpoets, but their poetic experience did not translate into greater retrieval success.

\section{First Letter}

Of the information available during TOTs, none has garnered more attention than the first letter of the target word. Subjects' high degree of accuracy in guessing the first letter has suggested an orthographic organization to long-term memory (R. Brown \& McNeill, 1966; Collins \& Loftus, 1975). This speculation is supported by the efficacy of experimenter-provided (Freedman \& Landauer, 1966; Gruneberg \& Monks, 1974) and subject-generated (Gruneberg, 1978; Reason \& Lucas, 1984) first-letter cues in accessing blocked TOT targets. Popularized accounts of TOTs use this fact to indicate that the target word is "just out of reach." An informal survey of memory-cognition texts (referred to earlier in this review) revealed that the majority (61\%) comment on the ready availability of the first letter of the target word during TOTs.

Subjects are correct in their first-letter guesses between 50 to $71 \%$ of the time: $50 \%$ (Rubin, 1975), $51 \%$ (R. Brown \& McNeill, 1966, pilot study), 57\% (R. Brown \& McNeill, 1966, main study), 59\% (Yarmey, 1973, famous persons' last names), 68\% (Yarmey, 1973, famous persons' first names), and $71 \%$ (Koriat \& Lieblich, 1974). Although these percentages seem impressive, it is important to consider chance guessing probabilities. All letters are not equally probable at the beginning of words, so a simple chance baseline ( 1 in 26) is inadequate. To establish a control comparison, Koriat and Lieblich (1974) told subjects to guess the first letter of the target while in a "don't-know" state. The guesses were correct only $10 \%$ of the time, considerably less than the percentages just listed.

Koriat and Lieblich (1974) suggested that letter-guessing accuracy may be partially attributed to knowledge about the population of words from which the targets have been drawn rather than knowledge of a particular target word. To illustrate their concern, they required subjects to generate 10 common and 10 uncommon words. The first-letter frequencies of TOT target guesses were more highly correlated with first-letter frequencies of the uncommon than the common word pool. Thus, it appears that a portion of the subjects' successful guesses during TOTs may be attributed to general knowledge about uncommon words.

Another measure of first-letter accuracy has been derived from SS words. Of SS words generated in a TOT, what percentage match the target on first letter? This derived analysis yields a correspondence rate similar to that found with direct guesses: 49\% (R. Brown \& McNeill, 1966), 58\% (Browman, 1978), 77\% (Yarmey, 1973, famous person's last names), and 83\% (Yarmey, 1973, famous person's first names).

Beyond first letter matches, how often does the first sound of the related words correspond to the target? Burke et al. (1991) discovered that the initial phoneme of related words matched the initial phoneme of the target $36 \%$ of the time, in contrast to a chance rate of $6 \%$ resulting from randomly matching related words to targets. Examining oral free associations during TOTs, Kohn et al. (1987) noted that $45 \%$ of all word fragments generated during TOTs matched the initial phoneme of the target word, which was greater than the $9 \%$ match rate when subjects were guessing.

In summary, subjects appear to be aware of the first letter or sound (phoneme) of the missing target word. This is evident from direct guesses as well as in words resembling the target in sound. General knowledge of the distribution probabilities of first letters in words may account for some correct guesses, but the accuracy rates appear to be consistently higher than chance.

\section{Additional Letter Positions}

The first letter of the target word has received considerable scrutiny because it is spontaneously reported more often than the others during TOTs (Lovelace, 1987). However, several studies have assessed subjects' knowledge of additional letters of the target word. R. Brown and McNeill (1966) inferred knowledge of noninitial letters from SS words generated during TOTs. They evaluated how often letters in each of the first three positions and last three positions of SS words duplicated letters in the same positions of the target word, considering only target words at least six letters long. A chance control comparison involved the percentage of letter position matches between SM and target words. This orthographic overlap of SS words to the targets is higher than for SM words in all positions except for the third from the last. The match is highest for the first part of the word, intermediate for the last part, and lowest for the middle, resulting in a U-shaped serial position function.

Rubin (1975) investigated whether subjects in a TOT have direct knowledge of letters other than the first one by having them generate letters from the missing target and place dashes to indicate missing letters. Rubin did not report the total number of letters correctly placed but presented a conditional analysis whereby a letter was only counted correct if the one preceding it was also correct. He did this analysis both in the left-toright (forward) and the right-to-left (reverse) directions. This analysis only allowed a direct assessment of the percentage of first $(50 \%)$ and last $(31 \%)$ letter correspondences, because probabilities for other letter positions were dependent on getting the first (or last) letter correct.

Rubin (1975) concluded that letters other than the first one are directly available in a TOT and that these letters are likely to be emitted in clusters corresponding to morphemes rather than syllables. The last-letter congruence was less than that found by R. Brown and McNeill (1966) for SS words, but this may be due to Rubin's restricted sample that consisted of the four words yielding the highest number of TOTs in R. Brown and McNeill's study: sampan, philatelist, Ebenezer, and ambergris.

Koriat and Lieblich (1974) extended the R. Brown and McNeill (1966) technique by asking subjects to guess last and middle letters of the target word (as well as the first) while in a TOT. They did not report middle letter matches, perhaps because their frequency was too low. Final-letter guesses were 
correct $69 \%$ of the time, which was above a $17 \%$ chance rate for subjects in a don't-know state. Koriat and Lieblich (1975) reanalyzed the data from their earlier study (Koriat \& Lieblich, 1974) using R. Brown and McNeill's technique to determine letter position overlap between SS words and corresponding targets. SS words correspond to the target word in five out of six letter positions, similar to the findings of Brown and $\mathrm{McNeill}$, but their outcome differs from R. Brown and McNeill's in that the SS words match letters at the end of the target more often than those at the beginning.

Koriat and Lieblich (1975) statistically removed the chance overlap probabilities for each letter position by superimposing the entire set of target words on the entire group of SS words. With this adjustment, the SS words match the target words in all six letter positions. Furthermore, the first letter correspondence was greater than the other five positions, with no difference among the remaining five. On the basis of this outcome, they emphasized that their uncorrected matches (Koriat \& Lieblich, 1974), as well as R. Brown and McNeill's (1966), are inflated for later positions because all words overlap more often by chance in their endings. Kohn et al. (1987) confirmed this observation. Taking words generated by subjects in a non-TOT (guess) state, the letters matched the end of the target about a third of the time and the initial portion on about $5 \%$ of the occasions.

In general, subjects in a TOT appear to have a moderate degree of awareness of the last letter, although this is less pronounced than first-letter knowledge. Subjects also seem to have some appreciation of the middle letters in the target, although this is considerably less than knowledge of the first and last letters.

\section{Number of Syllables}

Of all types of target word structural information, Lovelace (1987) discovered that the number of syllables was most likely to come to mind spontaneously during a TOT. R. Brown and McNeill (1966) directed their subjects to guess how many syllables were in the missing target word, using categories of one to five. Subjects were correct in $60 \%$ of their guesses in the main study and $47 \%$ in a pilot investigation. A separate analysis of targets of one through five syllables in length (from their main study) yielded syllabic guess accuracies of $53 \%, 67 \%, 65 \%, 27 \%$, and $25 \%$, respectively.

Koriat and Lieblich (1974) found that subjects were accurate on $80 \%$ of syllabic number estimates, and separate analyses on target words one through four syllables long resulted in accuracies of $74 \%, 91 \%, 78 \%$, and $62 \%$, respectively. This pattern was similar to R. Brown and McNeill's (1966), with the highest accuracy on two-syllable words and a decline in both directions from that category. Koriat and Lieblich's subjects may have been more accurate than R. Brown and McNeill's because they were not biased by an atypically high endpoint (5). Koriat and Lieblich cautioned against overinterpreting these data, because correct guesses in the don't-know state were also moderately high (38\%). Thus, much of the accuracy can be attributed to guessing strategies.

Yarmey (1973) also found subjects to be highly accurate in syllabic length guesses, correctly estimating $73 \%$ of first names and $79 \%$ of last names of famous persons. However, these figures become less impressive when one considers that most proper names are one or two syllables long. Rubin (1975) also asked subjects to guess the number of syllables in the target and reported that "two thirds" of the guesses were correct (exact percentage not provided).

The number of syllables in the target word can also be inferred from SS words. These matched the targets on $48 \%$ of occasions in R. Brown and McNeill's (1966) investigation, which contrasts sharply with the $20 \%$ correspondence for SM words. Burke et al. (1991) noted that 43\% of all words generated (SS and SM) during naturally occurring TOTs corresponded to the intended target in syllabic length, and Yarmey (1973) discovered a high rate of syllabic matches between SS names and targets: $83 \%$ for first names and $77 \%$ for last names.

Although information regarding number of syllables in the target word appears to be available to subjects in a TOT, chance guessing probabilities are high because of the narrow range of syllabic choices. Subjects are also aided in their estimates by simply knowing the general class of the target word. As Koriat and Lieblich (1974) pointed out, a medical term is more likely to be four syllables long than two, whereas an animal name is more likely to be two syllables than four.

\section{Syllabic Stress}

No TOT investigator has requested that subjects assess the syllabic stress of the missing target word, but this information has been inferred from SS words produced in a TOT. R. Brown and McNeill (1966) restricted their syllabic stress analysis to a small subset of TOTs (22\%) with multisyllable targets and SS words that match the number of syllables in the target. The overall correspondence was relatively high at $76 \%$. Of those targets with a stress on the first syllable, $81 \%$ of the SS words generated had a similar stress; of targets with a stress on the second syllable, $67 \%$ had the same pattern.

Combining data from R. Brown and McNeill's (1966) study and his own, Rubin (1975) examined syllabic stress accuracy as it related to accuracies in guessing the first or last letter of the target. Of $7 \mathrm{SS}$ words that matched the target on number of syllables and first letter, only 3 had the same stress pattern. In contrast, of 13 SS words matching number of syllables and last letter, 12 had the same stress pattern. Rubin proposed that an appreciation of syllabic stress is related to knowledge of the last, rather than the first, part of the missing target word. However, speculations based on a sample of only 20 SS words must be viewed with considerable caution.

In general, evidence for syllabic stress matches is meager and inflated by subjects' appreciation of natural linguistic probabilities.

\section{Target-Word Activation}

The fact that target-word information is available during a TOT strongly suggests that the target is in a state of partial activation. A more direct evaluation of target-word activation has involved both lexical decision and perceptual identification tests on the unretrieved TOT target word.

Yaniv and Meyer (1987) demonstrated that when subjects are 
in a TOT, their latency to subsequently identify the target word is reduced despite their inability to recall it. In their study, each block of trials consisted of two parts. A target word was first cued with a definition, and this was immediately followed by six lexical decision trials, which always included the target on one of the trials. When subjects in a TOT failed to retrieve the target word, their subsequent lexical decision to the target was faster than to a control word. Thus, it appears that a TOT may reflect an activation of the target word in the absence of retrieval. This activation persists up to several minutes and was also evident in subsequent recognition decision latencies.

Using a different approach to assess TOT target word activation, Naito and Komatsu (1989) used a perceptual identification task following the definition cue task. They found no difference in the identification probability for TOT, don't-know, and new words. Unfortunately, a substantial amount of time intervened between the initial target word block and the identification task. They waited until after all 50 definitions were attempted and then inserted a 2-min distractor task. Therefore, 5 to $10 \mathrm{~min}$ may have elapsed between the initial retrieval task and perceptual identification, making it possible that the target word activation had dissipated.

\section{Target-Word Familiarity}

These last two sections consider the objective frequency and categorical membership of the TOT target words. TOT probability traditionally has been assumed to be inversely related to normative frequency of the targets. "The words which induce tip-of-the-tongue states in us are, of course, the words we use but rarely in our everyday lives" (Ellis, 1985, p. 123). Such an assumption motivated R. Brown and McNeill (1966) to select target words with objective frequencies less than one per million but greater than one in four million (Thorndike \& Lorge, 1944), and most investigators have been guided by this logic. Some research, however, questions the validity of this assumption.

For instance, Yaniv and Meyer (1987) found TOT rates higher than R. Brown and McNeill's (1966), even though they used higher frequency targets. When subjects assess the familiarity of retrieved targets, the outcomes consistently indicate a high level of familiarity with the target word. Reason and Lucas (1984) discovered that after being retrieved, more TOT targets were rated very familiar (39\%) than either moderately familiar (35\%) or slightly familiar (27\%). Similarly, Cohen and Faulkner (1986) found that retrieved targets were rated well known $71 \%$ of the time and not very well known only $29 \%$ of the time, and subjects claimed to have had no trouble with recall prior to the TOT for $62 \%$ of the targets. Finally, Burke et al.s (1991) subjects rated target words as above average on a scale of very unfamiliar to very familiar. In these investigations of naturally occurring TOTs, most target words appear to be ones with which subjects are reasonably familiar. This suggests that TOTs may not be restricted to only the low ranges of target-word objective frequency. There are probably points on the frequency-familiarity dimensions above and below which TOTs are unlikely to occur, and it would be helpful to determine these boundaries. This could be accomplished by correlating TOT frequency with ob- jective target-word frequency across items in laboratory investigations.

\section{Target-Word Categories}

No comparison of different TOT-evoking potentials of various target-word categories has been made in the laboratory, but this question has been evaluated in diary research. Gruneberg et al. (1973) found that the majority of blocks were on the names of personal acquaintances $(40 \%)$, followed by geographical terms $(26 \%)$, political terms $(16 \%)$, and other $(18 \%)$. The high incidence of TOTs on persons' names $(66 \%)$ was confirmed by Burke et al. (1991), and this category broke down into personal acquaintances ( $28 \%$ ), famous persons $(19 \%)$, places $(12 \%)$, and movies, television, and books (8\%). The remaining TOTs were object names $(12 \%)$ and abstract words $(21 \%)$.

This propensity to block on proper nouns was also supported by Browman (1978). In a catalogue of TOTs experienced by herself and others, $50 \%$ were proper nouns, $25 \%$ were object names and abstract nouns, $14 \%$ were adjectives, and $9 \%$ were verbs. Cohen and Faulkner (1986) also found that the names of friends and acquaintances accounted for $69 \%$ of TOTs, and the names of famous persons precipitated another $17 \%$ of the blocks. The remaining TOTs were place names $(7 \%)$ and "other" $(8 \%)$. Cohen and Faulkner's data are biased because they instructed subjects to record details of name blocks, but their results still illustrate how many blocks are stimulated by familiar persons.

These investigations demonstrate that most naturally occurring TOTs arise from blocking on the names of friends, acquaintances, or proper names. Of course, this may simply be a reflection of how often these classes of information are accessed in daily routines. If TOTs are adjusted for the total number of retrievals of that particular word class, this may eliminate these apparent differences. Name TOTs may also be overreported because of their specificity. Blocks that occur with other categories of words may be readily sidestepped (and forgotten) by substituting a synonym. For instance, when a block occurs on the word acrimonious during routine conversation, one can quickly change to caustic and continue with hardly a disturbance in the discussion. In contrast, there is only one label that can be used for a particular person (Thomas Jefferson), place (Shanghai), or event (Thanksgiving). The name block commands more attention than the adjective block and is therefore more likely to be remembered later.

A systematic comparison of various categories within a laboratory study would be helpful in evaluating the differences reported in these anecdotal studies. A continuous generation procedure could be used, in which subjects retrieve items from a certain category until a TOT arises or the contents are exhausted (Gruneberg et al., 1973; Meyer \& Hilterbrand, 1984).

\section{Immediate Resolution}

The "fate" of TOTs will be addressed in the next two sections, including the likelihood of target-word recovery and techniques used to accomplish this. Resolutions are first examined within the "immediate" time frame, while the TOT experience is still active in consciousness. This usually spans several seconds to 
several minutes after the initial block. A TOT may also be resolved minutes, hours, or days after the TOT has left conscious awareness. These "delayed" resolutions will be addressed in the next section.

R. Brown and McNeill (1966) found that $41 \%$ of the target words were recovered while subjects were filling out the TOT questionnaire, which is probably within a minute or two of the initial blocking. Similar short-range recovery rates were also noted by Koriat and Lieblich $(1974 ; 41 \%)$ and Mitchell $(1983$; $42 \%$ ) while subjects were answering questionnaires about the block. Gardiner et al. (1973) noted that $40 \%$ of missing targets were successfully retrieved between 15 and $60 \mathrm{~s}$, and Finley and Sharp (1989) found that $48 \%$ of TOTs were resolved within 3 min by older adults. Yarmey (1973) provided resolution probabilities, but only on a subsample of TOTs in which subjects had guessed the famous person's "profession" or "last place seen." Of those TOTs, $59 \%$ were resolved within a minute or two. This may be an overestimate, however, because TOTs in which extraneous information is available may be relatively closer to resolution than those for which such information is not available.

Gruneberg et al. (1973) tape recorded subjects' commentary on their memory search and found that $66 \%$ of the TOTs were resolved within the session, with a median time between block and retrieval of $17 \mathrm{~s}$. About a third of the TOTs (36\%) were resolved within $10 \mathrm{~s}$, half by $20 \mathrm{~s}(54 \%)$, and three quarters by 40 $\mathrm{s}(77 \%)$. Subjects did persist, albeit at a declining rate, beyond 90 s (also see Gruneberg \& Sykes, 1978, for additional comments). Gruneberg et al. (1973) also examined how long subjects are willing to work at nonresolved TOTs before giving up. The median is $74 \mathrm{~s}$, suggesting that subjects show remarkable persistence in the pursuit of an unavailable word. In fact, over a third of the unsuccessful searches (34\%) were maintained past a minute and a half.

Using artificial materials (CVCs), Ryan et al. (1982) had subjects learn a list of paired associates and then tested them on response recall. Study time on the stimulus-response pairs during learning did not influence the TOT probability during later response recall, but it did affect the probability of response recovery when TOTs did occur. During a 20-s test interval, there were $8 \%, 10 \%$, and $16 \%$ responses recovered during TOTs from pairs in the 3-, 5-, and 7-s study conditions.

Finally, one diary investigation (Burke et al., 1991) required subjects to log how long it took to resolve TOTs. The range of times spanned several seconds to several days. Burke et al. presented a cumulative frequency distribution that indicates a recovery plateau at 1 to $2 \mathrm{~min}$ following the TOT. Assuming that this represents the break between immediate and delayed resolution (see Gruneberg et al., 1973), Burke et al. found an immediate resolution rate of about $30 \%$ for younger and midage adults, and $18 \%$ for older adults (estimates from Figure 1 of their study).

In summary, the probability that a target will be retrieved within a minute or two of the TOT varies around $50 \%$ in laboratory investigations. In addition, subjects appear willing to persist in retrieval efforts for several minutes. Recovery times are positively skewed, and artificial materials yield a lower recovery rate than natural materials. Naturally occurring TOTs seem to result in a lower immediate recovery rate of between 20 and
$30 \%$, although these data are less precise because they depend on retrospective self-reports.

\section{"Levels" of TOT}

R. Brown and McNeill (1966) made a distinction between two levels of TOTs: a "nearer" TOT in which the target word was recalled during the TOT, and a "farther" TOT when the target was not produced before the experimenter provided it. This labeling was intended to reflect that subjects provide more accurate target-word information in nearer, in comparison with farther, TOTs. For example, $62 \%$ of first-letter guesses were correct in nearer TOTs, and $42 \%$ were accurate in farther TOTs.

Koriat and Lieblich (1974) also discovered that subjects were more accurate across several measures in assessing target-word attributes when in a nearer TOT. Correct percentages during nearer versus farther TOTs, respectively, were as follows: first letter, $79 \%$ versus $35 \%$; last letter, $53 \%$ versus $47 \%$; and number of syllables, $92 \%$ versus $65 \%$. Similarly, Kohn et al. (1987) found differences on the percentage of oral responses that were lexically appropriate words $(82 \%$ vs. $76 \%)$, correct syllabic matches between target and generated words (35\% vs. $20 \%$ ), phonological correspondence of generated fragments to target $(80 \%$ vs. $48 \%$ ), matches of root morphemes of related words to targets ( $52 \%$ vs. $29 \%$ ), and matches of root morphemes of fragments to targets ( $46 \%$ vs. $16 \%$; nearer vs. farther TOTs, respectively).

Yarmey (1973) noted that correct assessments of a famous person's "profession" $(97 \%$ vs. $91 \%)$ and "place last seen" $(98 \%$ vs. 95\%) during TOTs differed between nearer and farther TOTs, respectively. These differences are in the direction consistent with the other investigations, but ceiling effects reduce the magnitude and interpretability of these differences.

These studies indicate that the information provided by subjects is more accurate when they are in nearer TOTs than when they are in farther TOTs, but a causal interpretation remains ambiguous. When subjects happen to retrieve accurate partial information, it may push them closer to the target word. On the other hand, being closer to retrieving the target word may make accurate information more accessible.

\section{Search Strategy}

Subjects can apparently access a variety of dimensions about the target word during the TOT, but is there a particular order in which this information comes to mind? Yarmey (1973) asked subjects to record the order in which they filled out the TOT questionnaire items. In searching for famous names, subjects tended to first scan general, nonverbal categories before accessing specific structural information. They initially tried to identify the individual's profession, then the place where they last saw the person, followed by the last time they saw the person. Finally, subjects attempted to identify the structure of the name-number of syllables, first letter of the name, and so forth. It is difficult, however, to determine to what degree this search strategy was consciously directed by the subject or to what degree it reflects how information naturally comes to mind as a by-product of the TOT state. Also, it is unclear how 
this pattern would generalize to other types of target-word categories.

\section{Search Effort}

Introspectively, the TOT is a mentally demanding activity. One is consumed with the search for the missing target word, to the exclusion of other mental processes. Is there evidence to support this apparent effortfulness? Ryan et al. (1982) used a secondary task to measure processing capacity allocated to the TOT resolution. After studying paired associates, subjects were tested for response recall. On each trial, the subject indicated whether the response was (a) known and available, (b) known but temporarily inaccessible (TOT), or (c) not known (DK). This evaluation was immediately followed by a 20 -s digit probe task, after which subjects attempted to recall the response. Performance on the digit probe task was significantly worse after subjects claimed to be in a TOT, in comparison with a DK state. Ryan et al. argued that a TOT creates an attention-demanding situation and that "attentional capacity that is denied the number-probe task during 'TOT' trials is reallocated to covert retrieval efforts" (p. 144).

The difference between the TOT and DK trials, however, could be accounted for by rehearsal rather than subconscious retrieval effort. If TOTs were resolved during the 20-s interval, then subjects may have done poorly on the secondary task because they were holding the just-retrieved target in short-term memory. To test this, Ryan et al. compared number probe accuracy on TOT trials in which the target was produced versus was not produced after the distractor task. They found no difference in performance and concluded that the diminished attentional capacity is more likely due to subconscious retrieval efforts than differential rehearsal.

\section{Memory for Recalled TOT Targets}

What is the fate of target words that have been blocked? Are they more likely or less likely to be remembered in comparison with targets that were not blocked? Gardiner et al. (1973) investigated the relationship between the initial difficulty of word retrieval, defined by recall latency, and the probability of remembering the item on a subsequent recall test. A pilot study revealed that targets that took longer to generate were better remembered on a later test. However, when TOT items were excluded from the analysis, the relationship between initial retrieval time and subsequent recall probability was reduced.

This preliminary finding motivated their main study, in which they found that target words initially retrieved within 0 to $15 \mathrm{~s}$ were later recalled at a lower rate $(27 \%)$ than those retrieved between 15 to $60 \mathrm{~s}(47 \%)$. However, when these longer retrievals were separated into TOT and non-TOT types, the recall probability for non-TOT items was the same as items retrieved under $15 \mathrm{~s}(27 \%)$, whereas the recall of TOT items was much higher (59\%). Apparently, evoking a TOT, rather than retrieval time per se, was the key factor determining higher recall. Even when the target word was supplied by the experimenter, the difference was found: Eventual recall was $36 \%$ for non-TOT items and $49 \%$ for TOT items.
This effect may be due to the heightened attention a TOT target receives when it is eventually retrieved or provided. It is also possible that during the TOT, some of the target word's attributes are activated (first letter, syllabic composition, etc.), which leads to a richer episodic memory trace. Gardiner et al. (1973) acknowledged that it is impossible to test these explanations within the confines of their particular paradigm.

\section{Delayed Resolution}

After the TOT experience has dissipated, the target word may be recovered from several minutes to several days after the original block. These recoveries are usually assessed through diary records, recontacting subjects, or presenting memory cues again at a later time.

Gaining a clear picture of the probability that a TOT will eventually be resolved is difficult because time intervals, measurement procedures, and data reporting vary from study to study. When subjects trace their naturally occurring TOTs, Burke et al. (1991) discovered that nearly all $(96 \%)$ were resolved. Cohen and Faulkner (1986) found that over a third (38\%) of the TOTs were resolved within an hour, with some resolved several days later. Although "most" targets were eventually recalled, Cohen and Faulkner did not provide specific percentages. In laboratory investigations, Read and Bruce (1982) found that $74 \%$ of the TOT targets not recovered during the lab session were retrieved in the 2 days intervening between sessions. Examining a smaller time span, Gruneberg et al. (1973) noted that $6 \%$ of TOTs were resolved within $4 \mathrm{hr}$. In summary, substantial proportion of blocked target words appear to be recovered after the TOT experience, but the time course of this recovery or total number resolved is difficult to glean from these studies.

Another aspect of target-word recovery is the manner in which they come to mind: conscious search effort, inadvertent environmental cuing, and spontaneous retrieval. A lively debate in the TOT literature centers on the possibility of spontaneous target retrievals, or pop-ups (Reason \& Lucas, 1984). Folk wisdom suggests that after a nonresolved TOT, the target word often will unexpectedly come to mind at a later time, when one's attention is diverted elsewhere. Wood (1983) suggested that "the name just pops into awareness, sometimes immediately and at other times after minutes, hours, days, or even weeks" (p. 10), and Hintzman (1978) proposed that "sometimes the answer seems to come to us spontaneously, after some 'incubation' time" (p. 307). Reason and Mycielska (1982) commented that "two of us were struggling to recall the name of Andre Previn.. . . The TOT state continued for hours until the target popped up of its own accord" (p. 123). Finally, Norman and Bobrow (1976) described an experience in which one of them tried several techniques to coax a missing word to mind, and then gave up. The word finally popped up

1 hour and 39 minutes after the start of the recall attempt. The word came without hesitation. There was no doubt that it was correct. For the hour prior to the solution, there was no recollection of thought on the topic. (p. 116)

Research has evaluated the issue of delayed TOT resolution, including pop-ups. Gruneberg et al. (1973) contacted their sub- 
jects between 2 and $9 \mathrm{hr}(M=4.3 \mathrm{hr})$ after participating in a TOT experiment. All subjects had experienced unresolved TOTs during the experimental session. Four of 18 subjects reported resolving one TOT, accounting for $6 \%$ of their unresolved retrievals. All recoveries were a result of conscious search efforts. Gruneberg et al. recued subjects on the originally blocked targets, and over a fifth (22\%) were resolved by this second effort. These were resolved much faster than first-session recoveries. Nearly half $(47 \%)$ were retrieved within $10 \mathrm{~s}$ of the second inquiry, whereas about a third were resolved this quickly on the first try. No second-try resolutions took longer than $90 \mathrm{~s}$, in comparison with $11 \%$ of the first-session recoveries. Of course, these shortened solution times may reflect decreased motivation to search for the same elusive target words a second time.

In a more ambitious effort to track the fate of blocked target words, Read and Bruce's (1982) subjects participated in 11 test sessions spanning 19 days. Entertainers' names were cued either verbally or pictorially. Subjects were presented one type of cue until either a block occurred or 10 different cues had been presented, and then were switched to the other cue type. At the start of each session after the first, subjects described any target-word recoveries that had occurred between sessions. If a TOT had not been resolved, the subject was recued with either the same or the other type of cue (visual or verbal) mixed in with new target cues.

Of the resolutions, $61 \%$ were due to conscious mental efforts, $13 \%$ to external environmental clues (i.e., seeing the name in the newspaper), and 5\% to cues provided in a subsequent laboratory session. About one fifth (21\%) of the TOTs were never resolved. Read and Bruce (1982) found that the most common self-resolution strategies involve partial structural information (name length, sounds, or letters), followed by contextual information such as a person's profession, ethnic origin, or spouse's name. Visual and auditory mental images of the person's face, voice, or gestures were also used in an attempt to find the missing name, as was sorting through the category of names similar to the target person's. Few targets emerged spontaneously (3\%). Read and Bruce (1982) also reported an unpublished study with a comparably low percentage of pop-ups (5\%). They acknowledged that spontaneous retrievals may happen, but the actual incidence is overestimated because such retrievals are surprising and make a distinct imprint on memory.

Pop-ups may also be overestimated if the triggering memory event is quickly forgotten. As a series of thoughts and sensations pass through the mind during normal waking consciousness, a particular event may trigger the missing information but become quickly lost in the elation over the target-word recovery. An overt version of this inadvertent triggering was reported by Bolinger (1961), in which a subject blocked on the name of Al Capone's heroine. Several days later, while cycling, he mused to himself how beautiful it is to ride in the long "days in May," at which point the missing target immediately came to mind (Daisey Mae). Therefore, some of the incidence of pop-ups may be accounted for by forgotten cues.

Several naturalistic, diary investigations have yielded substantial rates of pop-ups. Reason and Lucas (1984) discovered that nearly a third (32\%) of the blocked target words come to mind spontaneously. Other TOTs were resolved by internal strat- egies (searching the alphabet or generating similar words), and external techniques (asking others or consulting an encyclopedia). Subjects also recorded the number of separate searches needed to retrieve the target word, and over half (55\%) required only a single search.

In another diary study, Burke et al. (1991) discovered that most TOTs $(54 \%)$ were resolved by pop-ups, with fewer resolved by a directed memory search and consulting books or other persons. Younger adults are more likely to engage in active retrieval strategies, whereas older adults are more passive and reported a larger percentage of pop-ups. Burke et al. suggested that older adults appear more confident about the likelihood of spontaneous retrieval than are younger adults.

In a more detailed analysis of the self-reported resolution times, Burke et al. (1991) found a linear relationship between the cumulative frequency of resolution and log time up to about 2 days, when nearly all TOTs had been resolved. Burke et al. found that mean resolution times varied as a function of the method used, with pop-ups taking the longest time, consulting external sources next longest, and direct memory searches the least time. TOTs in which related words occurred took reliably longer to resolve than ones in which no related word occurred.

In another naturalistic diary investigation, Cohen and Faulkner (1986) discovered that $26 \%$ of TOT targets were recovered by mental search efforts, $22 \%$ by external aids, and $17 \%$ through pop-ups ( $34 \%$ were unclassified). The resolution percentages for active searches (mental and external) were comparable to those found by Burke et al, whereas the likelihood of pop-ups was much lower.

Norman and Bobrow (1976) provided a possible resolution to the conflicting data on the likelihood of pop-ups. They suggested that both lower (perceptual) and higher (problem solving) levels of cognition can operate subconsciously through the activation of schemas. However, each process needs a driving force. For the lower systems, sensory input can provide the impetus, but the higher level operations need a period of concentrated effort to initiate the process. Therefore, a precondition for a subsequent pop-up is a period during which the subject concentrates on the missing word, recalling possible attributes and related words. Without this initial energizing, the subconscious process will not maintain itself.

This interpretation may help to resolve the discrepant findings with respect to pop-ups following the TOT experience. Investigations reporting higher rates of spontaneous recovery (Burke et al., 1991; Cohen \& Faulkner, 1986; Reason \& Lucas, 1984) have examined naturally occurring TOTs, whereas those with the smallest recovery rates (Gruneberg et al., 1973; Read \& Bruce, 1982) have focused on artificially induced TOTs. Perhaps the subjects' level of motivation and involvement in their self-generated memory blocks is much greater, resulting in a higher energizing of the subconscious search process not duplicated by artificial laboratory tasks.

\section{Etiology}

An intriguing aspect of the TOT is the initial memory "trip up," which propels one into a state of suspended retrieval. Reason and Mycielska (1982) suggested that minor perturbations of attention caused by distraction or preoccupation result in re- 
trieval "hiccups." On most occasions, the word is quickly recovered and the person barely notices the memory lapse, or microTOT state. Sometimes, however, these attentional blips lead the retrieval process to stall, demanding full conscious attention and resulting in a full-blown TOT. Such speculation is colorful but difficult to empirically verify.

Speculations on the cause of the TOT experience have centered around two positions: incomplete activation and blocking. With the incomplete activation viewpoint, the TOT represents a normal word-search process that has been drastically slowed. The word-finding activity is on course but becomes stuck in the vicinity of the target because of insufficient targetword information. In contrast, the blocking perspective suggests that the TOT represents a memory search that has become sidetracked in the wrong memory location.

\section{Incomplete Activation}

Under the incomplete-activation interpretation of the TOT, successful word retrieval requires an aggregation of information from various sources and requires that on occasion these sources may be insufficient to exceed the threshold for retrieval. This position was advocated by R. Brown and McNeill (1966), who used a computer punch card analogy to illustrate their viewpoint. A definition or cue directs the subject's attention toward a card on which the target word should be written. On some occasions, the word entry is incomplete or indistinct (i.e., only the first and last letters appear on it) but can still guide a second search based on this fragmentary information. The second retrieval accesses a batch of related words sharing these same fragmentary characteristics. For instance, if the target word is sextant, the subject may first locate an incomplete card containing "se--t." Using this information, the subject reenters the lexicon to retrieve words such as secant or sextet. This second search effort, primed by the partial information, acts to clarify the faint entry on the original card "the way heat brings out anything written in lemon juice" (p. 335). It may even provide another version of the word on a different card.

In a subsequent elaboration of this viewpoint, $R$. Brown (1970) suggested that when one reads words, one does not thoroughly process them on each exposure. Salient parts of the word, such as the first and last letters, are processed more completely than redundant portions, such as the middle letters. Therefore, the first and last parts are more likely to be associated with other words through repeated, contiguous experience. When attempting to retrieve these words later through related word cues, the strongly associated fragments (i.e., first letter) may be the only portions that become available to consciousness.

Under the incomplete-activation position, the availability of target-word information reflects a gradual narrowing of the memory search process. Wenzl (1932, cited in Woodworth, 1938) proposed that related words and target fragments provide guideposts along the retrieval route and suggested that the typical recall process is like a mental funnel, with recall proceeding from the general toward the specific. Broad characteristics of the word are activated first-its atmosphere, initial sound, and rhythm - followed by the specific word. The availability of general information about the target was referred to as "generic recall" by $\mathrm{R}$. Brown and McNeill (1966). Reed (1974) also viewed the TOT experience as reflecting a constructive process of retrieval:

The TOT experience suggests that we have activated one or more schemata correctly and that reconstruction is taking place. There is a good basis for our conviction that we have captured our item, even though it has not yet crystalized. (p. 86)

Kohn et al. (1987) used a continuous free-association procedure to evaluate this interpretation. If the TOT involves a gradual narrowing of focus on the target-word location, they reasoned that the emergence of fragmentary information should follow a systematic pattern with each successive piece of datum more related to the target word than the previous one. Subjects were encouraged to report any information that came to mind during a TOT, and of those TOTs with multiple pieces of information available (about a quarter of the total), less than 1\% reflected such a systematic narrowing (e.g., "n-, neo, nepotism," p. 251). Kohn et al. viewed this as evidence against the incomplete activation position, but such an interpretation assumes that subjects describe information in the order in which they think of it and that they provide everything that comes to mind.

In a recent elaboration of the incomplete activation hypothesis, Burke et al. (1991) suggested that the retrieval deficit occurs in the linkage between the phonological word systems and semantic word systems. In most retrievals, the meaning representation of the word allows direct and immediate access to its sound representation, thus enabling vocalization. However, on occasion the phonological entry is merely primed but not fully activated, instilling a certainty of knowing in the absence of production ability.

Burke et al.'s (1991) model is fairly complex and interwoven into language production theory. The details are not presented here, but several findings support their speculation. The preponderance of naturally occurring TOTs involve words that have not been frequently or recently activated, according to subjects' self-reports (Burke et al., 1989). This is what would be anticipated if the probability of a TOT was inversely related to the strength of the semantic-phonological connection for a word. Furthermore, Burke et al. speculated that the increase in TOT frequency with older adults results from the weakening of the semantic-phonological connection, as reflected in the reduction in ancillary information about the target word (first letter, number of syllables, related words) available to older adults.

\section{Blocking}

According to the incomplete activation position, the presence of words semantically and structurally related to the target reflects the level at which the search is stalled. The occurrence of related words is also central to the blocking position but is interpreted differently. A TOT reflects a memory search effort that took an inadvertent detour prior to the target and located an incorrect word. "In these cases of hampered recall, one seems to start towards the goal but to stray into a blind alley" (Woodworth, 1940, p. 127). This incorrect word diverts attention away from the target word and competes with it. A semantic or phonemic resemblance to the target makes the related 
word compelling, and efforts to retreat from it back to the target word become arduous.

As Freud (1901, cited in Reason \& Lucas, 1984) suggested, when we are searching for an elusive name, we frequently retrieve names that "although immediately recognized as false, nevertheless obtrude themselves with great tenacity" (p. 189). Some investigators reveal their bias by labeling these related words blockers (Burke et al., 1988; Reason \& Lucas, 1984) or interlopers (Jones, 1989; Jones \& Langford, 1987). To remain neutral on this issue, I use the term related words in this review (see Cohen \& Faulkner, 1986, for further comment).

Reason and Lucas (1984) discovered that subjects having TOTs rated more than three quarters of the related words higher in either frequency or recency than the target. This outcome suggested to Reason and Lucas that the related word may provide a more compelling destination near the target, diverting the search process toward it. They use a colorful analogy for their speculation, likening the related word to the "ugly stepsister" in the story of Cinderella. The prince (memory search) tries to find the correct word (Cinderella) to fit the definition (slipper), but the ugly stepsisters (related words) intercept the search effort. Reason and Lucas suggested that once these words are experienced, they tend to recurrently intrude into consciousness because recent experience makes them more likely to be reselected. This is similar to the process whereby words that have already been retrieved are more likely to be sampled again, owing to recent strengthening, thereby inhibiting the recall of the remaining category items (e.g., Rundus, 1973; Watkins, 1975). Schvaneveldt, Durso, and Mukherji (1982) also supported this explanation for the initiation and maintenance of TOTs.

A reasonable strategy to eliminate this blocking would appear to be consciously ignoring this related but incorrect word. As Woodworth (1938) suggested, "the wrong name recalled acquires a recency value and blocks the correct name" and "a rest interval allows the recency value of the error to die away" ( $p$. 38). Reason and Lucas (1984) argued that individuals hold onto the related word rather than discarding it because it generates a sense of closeness to the target and motivates them to continue the search. Burke et al. (1991) argued that the presence of related words should actually discourage subjects from pursuing the retrieval effort because activation is siphoned away from the target word. However, Burke et al. found no difference in subjects' confidence in eventual target word retrieval between TOTs with and without related words.

A major flaw with the blocking interpretation of TOTs is accounting for the substantial percentage of TOTs in which no related words are reported. Reason and Lucas (1984) suggested that subjects either start closer to the target on these occasions or that a clearly inappropriate word comes to mind that can be quickly rejected, avoiding the strengthening of repeated incorrect retrievals. It is also possible that "unconscious" alternative words come to mind that are too weak to be retrieved but are capable of inhibiting target retrieval (Burke et al., 1991). In any case, the blocking model is certainly weakened by the fact that TOTs are possible without conscious awareness of extraneous words.

To test the blocking against incomplete activation views, Jones and Langford (1987) manipulated the type of related- word primes that accompanied each definition: phonologically related, semantically related, both phonologically and semantically related, and neither phonologically nor semantically related to the target word. Under incomplete activation, retrieval should benefit from the availability of related words, and the number of TOTs should be reduced when related words are presented along with the definition. In contrast, providing the related words should increase TOTs from the blocking perspective. Jones and Langford found no difference as a function of semantic relatedness, whereas phonologically related primes yielded significantly more TOTs than phonologically unrelated primes. This provides evidence against the incomplete activation and for the blocking position (see Jones, 1988, for further comment).

Jones (1989) extended this research to make the correspondence between the target and phonologically related word more precise. Whereas only the first letter matched in Jones and Langford (1987), both the first letter and number of syllables overlapped in Jones (1989). Furthermore, Jones presented the related word either before or after the definition was read. Jones reasoned that with incomplete activation, a related word following the definition (at the start of retrieval) should facilitate target access (fewer TOTs) more than would a related word presented prior to the definition. The opposite should happen under the blocking position: A related word at the end of the definition should increase the probability of TOTs in relation to one preceding the definition.

Jones (1989) again discovered evidence for the blocking and against the incomplete activation hypothesis: Related words presented after the definition resulted in more TOTs than ones provided prior to the definition. In addition, he replicated Jones and Langford's (1987) finding that words phonologically related to the target caused significantly more TOTs than phonologically unrelated ones, and a semantic relationship between the target and related word had no effect.

Unfortunately, in the Jones studies (Jones, 1989; Jones \& Langford, 1987) related-word conditions were not balanced across targets. Instead, a particular set of target words received only phonetically related words, another only semantically related words, and so forth. Therefore, the effects of target-prime relationship cannot be separated from the differing propensities of particular target words to elicit TOTs. Burke et al. (1991) discussed another problem with these investigations (Jones, 1989; Jones \& Langford, 1987). After subjects experience several TOTs, they may discover that they have access to some structural information about the target. After this realization, when shown a phonologically related prime, they may believe that because they have some partial information about the target word, they could be in a TOT.

The role of related, but incorrect, words in causing and maintaining TOTs was also discussed by Roediger (1974) and A. S. Brown $(1979,1981)$. Roediger noted that there are many retrieval paradigms in which the presentation or retrieval of a word can actually suppress the recall of related words. He likened this to the TOT experience, in which word fragments or whole words may be a hindrance to eventual target word retrieval. A. S. Brown (1979) provided experimental support for such speculation. When a related word preceded the targetword definition, subjects found target-word retrieval more dif- 
ficult, as reflected in increased retrieval latencies and decreased retrieval probabilities, in relation to an unrelated prime. This effect apparently occurs only when the prime word is a target on some trials. When subjects know that the prime will never be the target, the inhibition disappears (Bowles, 1989; Roediger, Neely, \& Blaxton, 1983). This fits the dynamics of the TOT experience in the following manner. When the subject expects that a word could be the target, as in the TOT experience and in A. S. Brown (1979), it has the potential to hamper target retrieval. When subjects appreciate that the word presented cannot be a target (Bowles, 1989; Roediger, Neely, \& Blaxton, 1983), no inhibition occurs. Although they argued against the blocking hypothesis, Burke et al. (1991) did discover that target-word recovery took about three times longer when a related word was present during a TOT than when one was not. According to the incomplete activation position, recovery times should have been equivalent, or even shorter, when related words were present.

Miller (1979) espoused a theoretical position that incorporates this blocking position, suggesting that "successful recall ... depends to some extent on the ability to inhibit the retrieval of potential responses that are incorrect (i.e., other common words in the subject's vocabulary)" (p. 140). Warrington and Weiskrantz (1970) used the failure of this suppression mechanism, or disinhibition, to clarify amnesic patients' difficulty with word retrieval. Miller further suggested that occasional disinhibition in normal adults could produce TOTs and that a gradual increase in the magnitude of disinhibition could result in the increase in retrieval difficulties experienced by older adults.

\section{Individual Differences}

Research on the TOT experience has been extended to different groups of individuals. With children, the questions are, At what age do they first experience TOTs, and what is their awareness of these blocks? With older adults, the research focuses on whether TOTs increase with age and, if so, how this relates to possible memory decline. TOTs have also been examined with aphasic patients to determine whether it provides an appropriate model for their language dysfunction. Finally, investigations outside of the English-language culture have helped establish the generality of the TOT experience.

\section{Children}

Only a few investigations have examined TOTs in children. Wellman (1977) evaluated a broad range of memory-monitoring abilities in children from kindergarten through the third grade. He assessed children's feeling of knowing for unrecallable target words that were cued by pictures. Their spontaneous comments suggested that they were occasionally experiencing TOTs. When unable to recall a target word, subjects often volunteered partial information about the unrecallable target, such as fragments of it ("vi" for "violin"), words acoustically related to it ("fumbler" for "funnel"), longer words that subsumed it ("thermostat" for "thermos"), or words rhyming with it ("hair" for "bear"). These responses accompanied only about $1 \%$ of retrieval efforts at each grade level (kindergarten, first grade, and third grade).

Subjects' spontaneous comments also reflected that they were experiencing a TOT: "I know I know that"; "I know, I just can't remember"; "why can't I remember?" Such reactions occurred on $2 \%, 2 \%$, and $4 \%$ of the retrieval attempts for children in kindergarten, first grade, and third grade, respectively. These data suggest a developmental increase in TOTs, with an increase from the first to the third grade level. Wellman also noted that like adults, these young subjects often "became agitated and frustrated with their inability to recall the name" (p. 20).

Another investigation is a case study of a 2-year-old Dutch child who exhibited a word-finding difficulty strongly resembling the TOT experience in adults (Elbers, 1985). Using the transcripts of tape-recorded conversations, Elbers showed that the child retrieved an incorrect word and was aware that it was wrong but was incapable of finding the correct word. Furthermore, the incorrect word resembled the target in number of syllables, syllabic stress pattern, and first syllable rhyme. The block also occurred three times, on separate occasions, for the same word. Although limited in scope, this demonstration suggests that the TOT experience may occur in very young children.

\section{Older Adults}

A number of studies have examined TOTs in older adults, perhaps because older adults often express concern about such memory difficulties. With subjective estimates of TOT incidence, Sunderland et al. (1986) found a median of once a week, and Burke et al. (1991) found a comparable estimate of approximately 0.8 TOTs per week, which was essentially the same across younger, midage, and older age groups.

The actual incidence of TOTs was assessed by Burke et al. (1991) and Cohen and Faulkner (1986) using 4-week diary studies. Burke et al. found that the weekly average was lower for younger (1.0) than for either midage (1.4) or older (1.7) adults, which did not differ, whereas Cohen and Faulkner discovered no difference between younger (2.0) and midage (2.0) groups, but both were significantly lower than the older group (4.0). Ironically, although Cohen and Faulkner had subjects record only proper name TOTs, their subjects reported more TOTs than Burke et al.s. Perhaps having subjects focus on a subset of information enhances their ability to remember TOTs by providing more specific retrieval cues. Sunderland et al. also found a TOT frequency (median) of two per week in a 1-week diary study for older adults, suggesting that Burke et al.'s outcome may be more representative.

Although Burke et al. (1991) and Cohen and Faulkner (1986) discovered more TOTs in older than younger subjects, this increase occurred by midage for Burke et al. but not for Cohen and Faulkner. No significant differences in verbal ability among groups were found in either study, but the trend may relate to the TOT differences. For Burke et al., the Wechsler Adult Intelligence Scale vocabulary scores were $67.1,72.6$, and 71.0 across younger, midage, and older groups, respectively. These means were $67.4,68.9$, and 73.3 for younger, midage, and older subjects (respectively) for Cohen and Faulkner. If verbal ability is 
positively related to TOT probability, then this pattern may clarify why the midage group resembled the older group in Burke et al. and the younger group in Cohen and Faulkner.

Further evaluation of Burke et al. (1991) and Cohen and Faulkner (1986) will exclude the midage groups because this usually duplicated that noted earlier: For Burke et al. the midage and older subjects were similar, and for Cohen and Faulkner, midage and younger subjects were comparable. The following age differences were consistent for both Burke et al. and Cohen and Faulkner: (a) Older adults gave higher familiarity ratings to target words, (b) unrelated words occurred more frequently during TOTs for younger adults, and (c) more target word information was available during the TOT (i.e., first letter and number of syllables) for younger subjects.

Several outcomes differ between the studies. TOTs are resolved more quickly by older adults in Cohen and Faulkner (1986), whereas Burke et al. (1991) discovered the opposite. On method of resolution, Burke et al. found that active strategies were used more often by younger subjects, whereas spontaneous retrievals occurred at a higher rate with older subjects. Cohen and Faulkner found no significant age difference for either type of resolution, although age trends resembled those of Burke et al.

Burke et al. (1991) discovered that proper names were the source of most TOTs for both younger and older adults, which confirms Cohen and Faulkner's (1986) wisdom in focusing on this category. Burke et al. did note a higher percentage of object word TOTs for older adults, whereas younger adults experienced considerably more TOTs involving nonobject words. They point out that this word class difference may result from younger (college student) subjects experiencing TOTs with academic materials, which are more likely to involve abstract (nonobject) terms.

In general, the aging research suggests that older adults are more prone to TOTs on a daily basis, have fewer target-word fragments and related words come to mind during the TOT, and take a less active role in resolving the TOT. Burke et al. (1991) proposed two factors to account for this TOT increase with age. If less recently experienced targets are more likely to elicit TOTs, then older adults will have more TOT-prone items in their memory store than younger adults. Burke et al.'s (1991) subjects' assessments of recovered TOT targets support such speculation. The other factor accounting for age differences may be a decline in the efficiency of the semantic to phonological word systems linkage, resulting in an increase in occasions in which the word's meaning is known but its speech production is blocked. This idea is supported by the decrease in availability of target-word information in older adults.

Age differences in TOTs need to be cautiously interpreted because of possible reporting criterion differences. Interestingly, Burke et al. (1991) found that older adults underestimated and younger adults overestimated their actual TOT frequency, suggesting a reporting bias in the opposite direction of that which might be inferred from objective group differences. Controlled laboratory research on TOTs comparing younger and older adults is needed to more systematically evaluate this possible age difference.

\section{Aphasic Subjects}

Aphasic subjects routinely experience word retrieval difficulties similar to those suffered by normal subjects in a TOT (Bruce \& Howard, 1988; Ellis, 1985). In picture naming, Barton (1971) noted that when unable to recall a target word, aphasic subjects still can supply generic information about the target. For each inaccessible target word ( $22 \%$ of retrievals), Barton requested that subjects indicate its initial letter, number of syllables (1 through 5), length (small, medium, or big), and the first related word to come to mind. The first letter was correct $62 \%$ of the time, a percentage significantly above chance and in the range of values obtained for normal adults. Furthermore, 13 of 16 subjects provided related words of similar meaning, and 9 subjects generated words similar in sound. Guesses of syllabic length were correct $72 \%$ of the time, and estimates of target word length were correct on $65 \%$ of the occasions.

Barton (1971) pointed out that aphasic subjects seem to know many generic properties of a word but cannot integrate them to produce the word, and he illustrates this struggle in one of his subjects, who claimed that

he always knows the word, and that he has been trying to tell us
that he knows these words, but that, for some reason, they just
don't come out. He was, incidentally, quite heartened by this op-
portunity to demonstrate this very fact. (p. 81 )

Extending Barton's (1971) investigation, Goodglass, Kaplan, Weintraub, and Ackerman (1976) differentiated among four types of aphasia: Broca's, Wernicke's, anomic, and conduction. As with Barton's subjects, Goodglass et al.'s subjects named object drawings and when faced with a retrieval failure provided the following concerning the target word: sound, initial letter, number of syllables, and an associated word.

Goodglass et al. (1976) discovered that subjects with conduction aphasia correctly predicted both the number of syllables (34\%) and first letter (34\%) significantly more often than either the subjects with Wernicke's (14\% syllables correct and $13 \%$ first letter correct) or anomic aphasia (10\% syllables correct and $6 \%$ first letters correct). Subjects with Broca's aphasia did not differ from the other groups (20\% syllables correct and $21 \%$ first letter correct). Unfortunately, these percentages are low and close to chance guessing probabilities (Koriat \& Lieblich, 1974).

It is difficult to interpret these data from aphasic subjects. Certainly, there is empirical ambiguity; Barton's outcome suggests some partial knowledge, whereas Goodglass et al's outcome does not. Beyond this, simply establishing a parallel between the features of aphasic subjects' typical nonrecall state and the TOT experience does not help clarify either phenomenon.

\section{Other Cultures}

Most literature on the TOT experience pertains to Englishspeaking subjects. Do persons in other cultures experience TOTs? Although one of the earliest systematic investigations of TOTs was by Wenzl (1932 as cited in Blumenthal, 1977) in Germany, there exists little additional literature on the cultural diversity of the phenomenon. Two exceptions involve Japanese subjects. Murakami (1980) discovered that a TOT experience 
accompanied $4 \%$ of retrieval efforts and that subjects correctly guessed the target's first letter $94 \%$ of the time and the last letter $59 \%$ of the time while in a TOT. In comparison with Englishlanguage studies, the TOT incidence is lower and the percentage of correct target-letter guesses is higher. Naito and Komatsu (1989) found a considerably higher TOT incidence of $26 \%$. However, this difference is difficult to interpret because they used a more liberal TOT criterion than did Murakami (1980).

Detailed comparisons with English-language research is problematic because of cultural differences in the language structure, but these studies support the fact that the TOT experience is generalizable across cultures.

\section{Related Phenomena}

This section of the review describes three phenomena related to the TOT experience: retrieval blocks involving different sensory domains, oral language production deficits, and written language errors.

\section{Extensions to Other Senses}

The expression "tip of the tongue" was originally coined as a vivid image to portray the physical feeling of a word on the verge of being spoken. This imagery has been extended to two other senses to describe the sensation that information is close to being expressed or perceived.

Erdelyi and Kteinbard (1978) investigated the phenomenon of hypermnesia, or the increase in recall performance over repeated testing without intervening study trials. As subjects recalled specific pictures, they occasionally had a tip-of-the-eye (TOE) experience on a picture, in which their mental image is "out of focus" and incapable of successfully cueing a target word. As one subject described it,

the most interesting subjective experience was getting a general
"visual feeling" in my mind for a particular shape such as length or
roundness. I remember seeing a vague, oblong shape in my mind
from which I was able to extract such items as gun, broom and
baseball bat; from an oval shape-football and pineapple; from
an inverted cup form-bell, funnel, and bottle. . . Just before
many of these recoveries, I often experienced what might best be
described as a "tip-of-the-eye" (TOE) phenomenon, in which I
was certain a particular item was on the verge of recovery but
which would take its time before suddenly coalescing into an
image in consciousness. (p. 280 )

The TOE resembles the TOT experience because fragmentary information concerning the target image precedes the clearly detailed image. Information on the TOE is not provided by Erdelyi and Kleinbard (1978), but it does appear to be reliable. They reported a second study in which several more subjects reported the experience during postexperimental questioning. This propensity for an image to come to mind prior to the word was also found by May and Clayton (1973). After presenting definitions of picturable objects, they found that $18 \%$ of the time, some aspect of the visual appearance was available prior to the name. This experience was not described as a TOE by May and Clayton, and it is probably different from the spontaneous TOE described by Erdelyi and Kleinbard.
However, it does demonstrate that imaginal fragments of the target can precede name recall.

Turning to a different sense, Lawless and Engen (1977) studied subjects' ability to name odors and form associations to them (also see Engen, 1987). Subjects would occasionally have a strong feeling of familiarity for the odor name without being able to recall it. An association to the missing odor name accompanied most of these blocks (83\%). Lawless and Engen labeled this the "tip-of-the-nose" (TON) experience and conducted an experiment modeled after R. Brown and McNeill (1966). TONs occurred on $6 \%$ of the retrieval attempts and were elicited by the majority ( 27 of 48 ) of odors used.

The TONs differ dramatically from TOTs in the types of partial information available. Subjects had difficulty accessing structural aspects of the odor name, such as SS words, number of syllables, syllabic stress, and letters. Only two correct responses were produced across 37 TONs. In contrast, subjects easily provided general categorical or imaginal information: similar odors (49\%), general category of the smell $(65 \%)$, object origin $(49 \%$ ), place origin $(49 \%)$, visual image of the object $(32 \%)$, and visual image of the place (24\%).

Subjects in a TON judged which of two odors were closer to the blocked target (e.g., mint and roses for the target cloves), and their selections corresponded to independent judges' choices $77 \%$ of the time. When subjects were provided with a dictionary definition, the TON target-word recovery rate was $70 \%$. The number of TONs resolved within the session can be inferred from the difference between the total and the number in which dictionary cues were provided. The short-term resolution rate of $38 \%$ is close to that found for TOTs (see the section Immediate Resolution in this review).

\section{Slips of the Tongue}

A slip of the tongue, or SOT, is when a word is inadvertently substituted in naturalistic speech production (i.e., "goof" for "golf," "psychotic" for "psychological"; Tweney, Tkacz, \& Zaruba, 1975). Collections of such naturally occurring errors have been analyzed for semantic and phonemic relationships to the intended target word (Fay \& Cutler, 1977; Tweney et al., 1975; Vihman, 1980; Zwicky, 1982). An SOT resembles a TOT in that both involve an error in word retrieval. With an SOT, related words come to mind but are not identified as such before output; with a TOT, related words may come to mind but are detected as incorrect before being spoken. Perhaps the key distinction between TOTs and SOTs is the level of monitoring during word production. When monitoring is low, the incorrect word is spoken and an SOT occurs. When awareness is higher, incorrect information is withheld, and a TOT results (see Ellis, 1985, for a further discussion of the relationship between SOTs and TOTs).

The majority of SOTs match the target in sound, considering an SS match as sharing at least one letter with the target in the same position: $86 \%$ (Tweney et al., 1975), 81\% (Fay \& Cutler, 1977), and 55\% (Dell \& Reich, 1981). This is comparable to $R$. Brown and McNeills (1966) finding that related words were predominantly SS during TOTs. The correspondence between syllabic number of SOT and target word ranges between $71 \%$ 
and $86 \%$ across studies (Fay \& Cutler, 1977; Tweney et al., 1975; Zwicky, 1982). Again, this is similar to the degree of overlap found between SS words and targets in TOTs (R. Brown \& McNeill, 1966). Syllabic stress matches were also extremely high between SS word and target, at over $90 \%$ for Zwicky (93\%) and Fay and Cutler (98\%).

On specific letter matches, Tweney et al. (1975) found that SS SOT words matched the targets $52 \%$ of the time on the first letter, $49 \%$ on the second letter, $62 \%$ on the second to last letter, and $77 \%$ on the last letter (estimated from their Figure 1, p. 393). Letter matches between SM SOTs and intended targets were much lower at $31 \%, 31 \%, 20 \%$, and $27 \%$, for first, second, second to last, and last letters, respectively. The SS words matched their targets better on the end position than on the beginning position, an outcome opposite of SS word matches in a TOT (R. Brown \& McNeill, 1966). Two other studies, however, found much higher first-letter correspondence rates of over 80\% (Vihman, 1980; Zwicky, 1982).

These SOT investigations indicate that SOTs are clearly related to intended targets and that this correspondence is higher than that between SS words and targets during TOTs. SS SOTs occurred more often than SM SOTs, the syllabic match rate of SS SOTs always exceeded 70\%, syllabic stress match was extraordinarily high, and the individual letter matches were consistently high across positions. Although this outcome does not imply that the two phenomena have the same underlying cause, the parallels warrant further comparisons.

\section{Slips of the Pen}

Another language production error that resembles the TOT experience is inadvertent word substitutions while writing. In analyzing 847 slips of the pen (SOP) on students' written applications to college, Wing and Baddeley (1980) discovered that the letter-position correspondence between the SOP and the intended target was similar to that between SS words and target words during a TOT. The percentage of matches was high across the five positions they compared, ranging from $70 \%$ to $90 \%$, and were greatest at the initial $(89 \%)$ and final $(90 \%)$ letter positions. This U-shaped serial position function for letter overlap was also noted by Hotopf (1980), who combined several different collections of SOPs. He found a range of correspondence between $66 \%$ and $93 \%$, with the highest overlap on the first-letter position (93\%) and the last-letter position (89\%; estimated from his Figure 1, p. 302).

These overlap percentages between the SOP and target word are higher than between SS words and a target word in the TOT, but the similar serial position function suggests underlying communalities between written and oral output errors. Hotopf (1980) increased the SOP incidence by requiring students to write hastily on a controversial topic in a noisy, distracting environment. The error rate doubled, implying that word selection or output errors (TOTs, SOTs, and SOPs) may result from distraction or misdirected attention. A test of this idea might involve making subjects perform a secondary task (i.e., visual monitoring) during retrieval to determine if this increases the TOT probability.

\section{Summary and Evaluation}

Despite the diversity of paradigms and analytical procedures, the TOT literature has produced some consistent findings, which are summarized in the following section. After this is a brief review of those issues that have yielded inconsistent or inconclusive outcomes.

\section{Consistent Findings}

1. The TOT phenomenon appears to be a nearly universal experience. Most subjects report such memory blocks in anecdotal reflection, diary studies, and laboratory investigations. Even when the TOT incidence is low (i.e., Mitchell, 1983) most subjects report them. The experience appears to span all ages, from elementary school children (Wellman, 1977) to older adults (Burke et al., 1991; Cohen \& Faulkner, 1986).

2. A variety of stimulus materials can elicit TOTs. Although definitional cues are the mainstay of this research (R. Brown \& McNeill, 1966), TOTs also occur with faces (Read \& Bruce, 1982; Yarmey, 1973), simple line drawings (Mitchell, 1989; Wellman, 1977), nonsense syllable pairs (Ryan et al., 1982; Wearing, 1987), and odors (Lawless \& Engen, 1977).

3. TOTs are reported to occur in daily life about once a week and to increase with age. In the lab, TOTs appear to occur on about $10 \%$ to $20 \%$ of all attempts to retrieve low-frequency target information.

4. Most naturally occurring TOTs are triggered by names of personal acquaintances, followed by names of famous persons and objects.

5. Words related to the sought-after target come to mind on between $40 \%$ and $70 \%$ of TOTs. This probably depends, to some extent, on how much the instructions suggest the occurrence of related words. Words both semantically and orthographically similar to the target are thought of, but orthographically related words predominate.

6. Subjects in a TOT correctly guess the first letter of the missing target word about $50 \%$ of the time, a figure that is well above chance. Indirect evidence of first-letter knowledge is also revealed in the first-letter correspondence between SS words and their intended targets.

7. The last letter of the target word also seems available during a TOT, as evidenced by direct letter guesses (Rubin, 1975) and inferences from SS words (R. Brown \& McNeill, 1966). Knowledge about other target letters (i.e., second and second from last) is limited.

8. Information on a target word's syllabic structure is available in a TOT as inferred by subjects correctly guessing the number of syllables $50 \%$ to $80 \%$ of the time. Chance guessing probabilities, however, account for a substantial portion of this because subjects not in a TOT correctly guess the number of target-word syllables $38 \%$ of the time (Koriat \& Lieblich, 1974).

9. Around one half of all TOTs $(40 \%$ and $66 \%)$ are resolved within a minute of the blocking experience.

10. TOT target words are recalled later at a higher rate than non-TOT targets. This difference is true whether subjects retrieve the target or have it supplied by the experimenter. 


\section{Ambiguous Findings}

1. The TOT experience often is associated with an emotional reaction or agitation (R. Brown \& McNeill, 1966). This is diffcult to quantify or assess, and there are no objective data on this issue. Some use an emotional reaction as a defining quality of a TOT (Gruneberg et al., 1973), and perhaps some measure of physiological arousal during retrieval, such as pupil dilation and galvanic skin response, might be useful in future research (Headley, 1981).

2. R. Brown and McNeill (1966) originally assumed that only low-frequency targets elicit TOTs. Most subsequent investigations have accepted this idea, but empirical verification of it is lacking. Variation in objective frequency of target words across studies has had little impact on TOT frequency, and subjects tend to rate blocked targets as relatively familiar (Burke et al., 1991; Cohen \& Faulkner, 1986; Reason \& Lucas, 1984). This issue could be resolved by an item-based correlation of TOT frequency with objective frequency, using a sample of target words spanning a broad range of objective frequency.

3. Does the TOT experience reflect a retrieval process stalled because of incomplete target activation or a retrieval sidetracked because of incorrect information? Neither position has received an adequate test, mainly because of the difficulty of experimental control with TOTs. This theoretical issue pivots on the role of related words in the TOT experience. Although R. Brown and McNeill (1966) suggested that related words faciliate eventual retrieval, others argued that they precipitate and maintain the target-word retrieval blockage (A. S. Brown, 1979; Jones \& Langford, 1987; Roediger, 1974).

4. The existence of later spontaneous retrievals (pop-ups) of blocked TOT targets has been supported in some investigations but not in others. The key distinction appears to be the manner in which the TOT is induced. Naturally occurring TOTs yield a moderate number of pop-ups ( $17 \%$ to $41 \%$ ), whereas studies that induce TOTs in the lab yield a much smaller percentage (5\% or less).

\section{Directions for Future Research}

The TOT phenomenon has intrigued researchers for nearly a century. R. Brown and McNeill (1966) broke the ground in the systematic investigation of this phenomenon, and most subsequent studies have modeled their materials or procedure, to some extent, after the original investigation. However, each study differs in the stimulus materials, questionnaire format, and summary statistics, making it difficult to directly compare outcomes among investigations.

A more comprehensive and consistent disclosure of certain types of information concerning TOTs must become routine if understanding of the TOT experience is to advance. A frequency distribution of TOTs for both subjects and target words should be standard. The total number of both successful and unsuccessful retrievals would help to evaluate the percentage of TOTs more clearly. A count of both positive (target word same as experimenter's) and negative (target word different from experimenter's) TOTs should be provided, even when negative TOTs are not used in the analyses. Subjects should be carefully instructed as to what is and what is not a TOT before participating in the study, and they should be asked to describe a personal TOT experience to verify that they can separate it from routine recall failure (Burke et al., 1991).

The scope of TOT research should be expanded on both subjects and materials. TOTs have been studied in children and older adults, but systematic laboratory investigations are lacking at each end of the age spectrum. Definitions of rare words and pictures of famous individuals have been heavily relied on to trigger TOTs. Studies suggest that paired associates may be effective in evoking TOTs (Ryan et al., 1982; Wearing, 1970). Pictured objects also yield TOTs (Mitchell, 1989; Snodgrass \& Vanderwart, 1980; Wellman, 1977) but have never been used in a study directly examining the TOT experience.

There is some concern that the instructions given to subjects determines the type of information produced during the TOT (cf. Gruneberg, 1978). For instance, R. Brown and McNeill (1966) instructed subjects to provide such structural features as the first letter and the number of syllables of the target word while in a TOT. This directive is problematic from two perspectives. First, it intimates that such information should be routinely accessible while in a TOT. The questions then become, What information comes to mind of its own accord, and what can be generated by the subject if asked to do so? Perhaps this is an unavoidable bind in studying covert behaviors: To collect systematic data, one must advise subjects concerning the possible range of responses, but in so doing one may artificially generate the appearance that such responses naturally occur.

A second problem with these instructions is biasing subjects toward providing acoustically or orthographically related words or information about the target word (see Dale \& McGlaughlin, 1971; Norman, 1969). Yarmey (1973) expressed a broader concern over the verbal bias in instructions and expanded his questionnaire to capture nonverbal and imaginal information about the inaccessible name of a famous person: profession, last place seen, and recency of last exposure.

Lovelace (1987) addressed the issue of questionnaire bias by using open-ended instructions to subjects in a TOT: "Perhaps you can tell me certain things about this word that you are looking for, or perhaps you can tell me something that the missing word reminds you of " (p. 371). Subjects generated an average of 2.7 attributes per TOT and volunteered the following types of target-word information: contextual attribute (32\%), descriptive attribute $(20 \%)$, number of syllables $(17 \%)$, word length $(14 \%)$, country or language $(14 \%)$, first letter $(12 \%)$, frequency of usage $(11 \%)$, whole word sound $(7 \%)$, time or era $(3 \%)$, partial recall (4\%), last letter (2\%), beginning sound (2\%), and ending sound (2\%). Lovelace, however, found this exercise problematic and lamented that "placement of the students' remarks into categories was not simple and clearcut, and agreement among the five judges was not as high as one might like" (p. 372).

Despite this limitation, the distribution of free-form responses suggests that the R. Brown and McNeill (1966) questionnaire may underrepresent the rich diversity of information available in a TOT. A variety of contextual, associational, and imaginal characteristics of the word may come to mind, and a broader questionnaire such as Yarmey's (1973) may better cover the complete spectrum of target-word information.

Supporting this speculation, Whitten and Leonard (1981) 
noted that when recalling the names of school teachers, target information (i.e., imagery) other than orthographic features were more readily available to subjects before retrieving the target name. Reaching back to the roots of TOT research, Wenzl (1932, cited in Blumenthal, 1977) included a wider series of queries for subjects to respond to, including affective reactions about the missing target word ("sad," "gloomy," "pleasing") and synesthetic associations to the target word ("bright," "sonorous"). Examining this earlier research may provide a paradigmatic break from a narrow focus on the structural characteristics of the missing target word.

\section{References}

Barton, M. I. (1971). Recall of generic properties of words in aphasic patients. Cortex, 7, 73-82.

Blumenthal, A. L. (1977). The process of cognition. Englewood Cliffs, NJ: Prentice-Hall.

Bolinger, D. L. (1961). Verbal evocation. Lingua, 10, 113-127.

Bowles, N. L. (1989). Age and semantic inhibition in word retrieval. Journal of Gerontology, 44, 88-90.

Browman, C. P. (1978). Tip of the tongue and slip of the ear: Implications for language processing. Unpublished manuscript, University of California, Los Angeles.

Brown, A. S. (1979). Priming effects in semantic memory retrieval processes. Journal of Experimental Psychology: Human Learning and Memory, 5, 65-77.

Brown, A. S. (1981). Inhibition in cued retrieval. Journal of Experimental Psychology: Human Learning and Memory, 7, 204-215.

Brown, R. (1970). Psychology and reading: Commentary on chapters 5 to 10 . In H. Levin \& J. P. Williams (Eds.), Basic studies on reading (pp. 164-187). New York: Basic Books.

Brown, R., \& McNeill, D. (1966). The "tip of the tongue" phenomenon. Journal of Verbal Learning and Verbal Behavior, 5, 325-337.

Bruce, C., \& Howard, D. (1988). Why don't Broca's aphasics cue themselves? An investigation of phonemic cueing and tip of the tongue information. Neuropsychologia, 26, 253-264.

Burke, D., MacKay, D. G., Worthley, J. S., \& Wade, E. (1991). On the tip of the tongue: What causes word finding failures in young and older adults? Journal of Memory and Language, 30, 237-246.

Burke, D., Worthley, J., \& Martin, J. (1988). I'll never forget what's-hername: Aging and tip of the tongue experiences in everyday life. In $\mathbf{M}$. M. Gruneberg, P. E. Morris, \& R. N. Sykes (Eds.), Practical aspects of memory: Current research and issues (Vol. 1, pp. 113-118). New York: Wiley.

Cohen, G., \& Faulkner, D. (1986). Memory for proper names: Age differences in retrieval. British Journal of Developmental Psychology, 4, 187-197.

Collins, A. M., \& Loftus, E. F. (1975). A spreading-activation theory of semantic processing. Psychological Review, 82, 407-428.

Dale, H. C. A., \& McGlaughlin, A. (1971). Evidence of acoustic encoding in long-term memory. Quarterly Journal of Experimental Psychology, 23, 1-7.

Dell, G. S., \& Reich, P. A. (1981). Stages in sentence production: An analysis of speech error data. Journal of Verbal Learning and Verbal Behavior, 20, 611-629.

Elbers, L. (1985). A tip-of-the-tongue experience at age two? Journal of Child Language, 12, 353-365.

Ellis, A. W. (1985). The production of spoken words: A cognitive neuropsychological perspective. In A. W. Ellis(Ed.), Progress in the psychology of language (Vol. 2, pp. 107-145). Hillsdale, NJ: Erlbaum.

Engen, T. (1987). Remembering odors and their names. American Scientist, 75, 497-503.
Erdelyi, M. H., \& Kleinbard, J. (1978). Has Ebbinghaus decayed with time? The growth of recall (hypermnesia) over days. Journal of Experimental Psychology: Human Learning and Memory, 4, 275-289.

Fay, D., \& Cutler, A. (1977). Malapropisms and the structure of the mental lexicon. Linguistic Inquiry, 8, 505-520.

Finley, G. E., \& Sharp, T. (1989). Name retrieval by the elderly in the tip-of-the-tongue paradigm: Demonstrable success in overcoming initial failure. Educational Gerontology, 15, 259-265.

Freedman, J. L., \& Landauer, T. K. (1966). Retrieval of long-term memory: "Tip-of-the-tongue" phenomenon. Psychonomic Science, 4, 309-310.

Freud, S. (1901). The psychopathology of everyday life. New York: Penguin.

Gardiner, J. M., Craik, F. I. M., \& Bleasdale, F. A. (1973). Retrieval difficulty and subsequent recall. Memory \& Cognition, 1, 213-216.

Goodglass, H., Kaplan, E., Weintraub, S., \& Ackerman, N. (1976). The "tip-of-the-tongue" phenomenon in aphasia. Cortex, 12, 145-153.

Gruneberg, M. M. (1978). The feeling of knowing, memory blocks and memory aids. In M. M. Gruneberg \& P. Morris (Eds.), Aspects of memory (pp. 186-209). London: Methuen.

Gruneberg, M. M., \& Monks, J. (1974). 'Feeling of knowing' and cued recall. Acta Psychologica, 38, 257-265.

Gruneberg, M. M., Smith, R. L., \& Winfrow, P. (1973). An investigation into response blocking. Acta Psychologica, 37, 187-196.

Gruneberg, M. M., \& Sykes, R. N. (1978). Knowledge and retention: The feeling of knowing and reminiscence. In M. M. Gruneberg, P. E. Morris, \& R. N. Sykes (Eds.), Practical aspects of memory (pp. 189 196). San Diego, CA: Academic Press.

Hart, J. T. (1965). Memory and the feeling-of-knowing experience. Journal of Educational Psychology, 56, 208-216.

Hart, J. T. (1966). Methodological note on feeling-of-knowing experiments. Journal of Educational Psychology, 57, 347-349.

Hart, J. T. (1967). Memory and the memory-monitoring process. Journal of Verbal Learning and Verbal Behavior, 6, 685-691.

Headley, D. B. (1981). Pupillometric assessment of retrieval operations in factual long-term memory. Acta Psychologica, 49, 109-126.

Hintzman, D. L. (1978). The psychology of learning and memory. San Francisco, CA: Freeman.

Hotopf, N. (1980). Slips of the pen. In U. Frith (Ed.), Cognitive processes in spelling (pp. 287-307). San Diego, CA: Academic Press.

James, W. (1890). Principles of psychology. New York: Holt.

James, W. (1893). The principles of psychology: Vol. I. New York: Holt.

Jones, G. V. (1988). Analyzing memory blocks. In M. M. Gruneberg, P. E. Morris, \& R. N. Sykes (Eds.), Practical aspects of memory: Current research and issues (Vol. 1, pp. 215-220). New York: Wiley.

Jones, G. V. (1989). Back to Woodworth: Role of interlopers in the tip-of-the-tongue phenomenon. Memory \& Cognition, 17, 69-76.

Jones, G. V., \& Langford, S. (1987). Phonological blocking in the tip of the tongue state. Cognition, 26, 115-122.

Kohn, S. E., Wingfield, A., Menn, L., Goodglass, H., Gleason, J. B., \& Hyde, M. (1987). Lexical retrieval: The tip-of-the-tongue phenomenon. Applied Psycholinguistics, 8, 245-266.

Koriat, A., \& Lieblich, I. (1974). What does a person in a TOT state know that a person in a don't know state doesn't know. Memory \& Cognition, 2, 647-655.

Koriat, A., \& Lieblich, I. (1975). Examination of the letter serial position effect in the "TOT" and the "don't know" states. Bulletin of the Psychonomic Society, 6, 539-541.

Koriat, A., \& Lieblich, I. (1977). A study of memory pointers. Acta Psychologica, 41, 151-164.

Kozlowski, L. T. (1977). Effects of distorted auditory and of rhyming cues on retrieval of tip-of-the-tongue words by poets and nonpoets. Memory \& Cognition, 5, 477-481.

Lawless, H., \& Engen, T. (1977). Associations to odors: Interference, 
mnemonics, and verbal labeling. Journal of Experimental Psychology: Human Learning and Memory, 3, 52-59.

Lovelace, E. (1987). Attributes that come to mind in the TOT state. Bulletin of the Psychonomic Society, 25, 370-372.

Matison, R., Mayeux, R., Rosen, J., \& Fahn, S. (1982). "Tip-of-thetongue" phenomenon in Parkinson disease. Neurology, 32, 567-570.

May, J. E., \& Clayton, K. N. (1973). Imaginal processes during the attempt to recall names. Journal of Verbal Learning and Verbal Behavior, 12, 683-688.

Meyer, G. E., \& Hilterbrand, K. (1984). Does it pay to be "Bashful"? The seven dwarfs and long-term memory. American Journal of Psychology, 97, 47-55.

Miller, E. (1979). Memory and aging. In M. M. Gruneberg \& P. E. Morris (Eds.), Applied problems in memory (pp. 127-149). San Diego, CA: Academic Press.

Mitchell, D. B. (1983). Retrieval from semantic and episodic memory: Individual differences in young and old adults. Dissertation $\mathrm{A} b$ stracts International, 43, 2724B.

Mitchell, D. B. (1989). How many memory systems? Evidence from aging. Journal of Experimental Psychology: Learning, Memory, and Cognition, 15, 31-49.

Murakami, Y. (1980). On the memory unit within kana-letter and kanji-letter words in the tip of the tongue phenomenon. Japanese Journal of Psychology, 51, 41-44.

Naito, M., \& Komatsu, S. (1989). Effects of conceptually driven processing on perceptual identification. Japanese Journal of Psychology, 31, 45-56.

Nelson, T. O. (1984). A comparison of current measures of the accuracy of feeling-of-knowing predictions. Psychological Bulletin, 95, 109133.

Norman, D. A. (1969). Memory and attention. New York: Wiley.

Norman, D. A., \& Bobrow, D. G. (1976). On the role of active memory processes in perception and cognition. In C. N. Cofer (Ed), The structure of human memory (pp. 114-132). San Francisco: Freeman.

Read, J. D., \& Bruce, D. (1982). Longitudinal tracking of difficult memory retrievals. Cognitive Psychology, 14, 280-300.

Reason, J. T. (1984). Lapses of attention in everyday life. In R. Parasuraman \& D. R. Davies (Eds), Varieties of attention (pp. 515-549). San Diego, CA: Academic Press.

Reason, J. T., \& Lucas, D. (1984). Using cognitive diaries to investigate naturally occurring memory blocks. In J. E. Harris \& P. E. Morris (Eds.), Everyday memory, actions and absentmindedness (pp. 53-69). San Diego, CA: Academic Press.

Reason, J. T., \& Mycielska, K. (1982). Absent-minded? Englewood Cliffs, NJ: Prentice-Hall.

Reed, G. (1974). The psychology of anomolous experience. Boston: Houghton Mifflin.

Roediger, H. L. (1974). Inhibiting effects of recall. Memory \& Cognition, 2, 261-269.

Roediger, H. L., Neely, J. H., \& Blaxton, T. A. (1983). Inhibition from related primes in semantic memory retrieval: A reappraisal of Brown's (1979) paradigm. Journal of Experimental Psychology: Learning, Memory, and Cognition, 9, 478-485.

Rubin, D. C. (1975). Within word structure in the tip-of-the-tongue phenomenon. Journal of Verbal Learning and Verbal Behavior, 14, 392-397.
Rundus, D. (1973). Negative effects of using list items as recall cues. Journal of Verbal Learning and Verbal Behavior, 12, 43-50.

Ryan, M. P., Petty, C. R., \& Wenzlaff, R. M. (1982). Motivated remembering efforts during tip-of-the-tongue states. Acta Psychologica, 51, 137-147.

Schvaneveldt, R. W., Durso, F. T., \& Mukherji, B. R. (1982). Semantic distance effects in categorization tasks. Journal of Experimental Psychology: Learning, Memory, and Cognition, 8, 1-14.

Snodgrass, J. G., \& Vanderwart, M. (1980). A standardized set of 260 pictures: Norms for name agreement, image agreement, familiarity, and visual complexity. Journal of Experimental Psychology: Human Learning and Memory, 6, 174-215.

Sunderland, A., Watts, K., Baddeley, A. D., \& Harris, J. E. (1986). Subjective memory assessment and test performance in elderly adults. Journal of Gerontology, 41, 376-384.

Thorndike, E. L., \& Lorge, 1. (1944). The teacher's word book of 30,000 words. New York: Bureau of Publications, Columbia University.

Tweney, R. D., Tkacz, S., \& Zaruba, S. (1975). Slips of the tongue and lexical storage. Language and Speech, 18, 388-396.

Vihman, M. M. (1980). Phonology and the development of the lexicon: Evidence from children's errors. Journal of Child Language, 8, 239264.

Warrington, E. K., \& Weiskrantz, L. (1970). Amnestic syndrome: Consolidation of retrieval? Nature, 228, 628-630.

Watkins, M. J. (1975). Inhibition in recall with extralist "cues." Journal of Verbal Learning and Verbal Behavior, 14, 294-303.

Wearing, A. J. (1970). On the trace strength of responses varying in correctness. Psychonomic Science, 21, 226-227.

Wellman, H. M. (1977). Tip of the tongue and feeling of knowing experiences: A developmental study of memory monitoring. Child Development, 48, 13-21.

Whitten, W. D., \& Leonard, J. M. (1981). Directed search through autobiographical memory. Memory \& Cognition, 9, 566-579.

Wing, A. M., \& Baddeley, A. D. (1980). Spelling errors in handwriting: A corpus and a distributional analysis. In U. Frith (Ed.), Cognitive processes in spelling (pp. 251-285). San Diego, CA: Academic Press.

Wood, G. (1983). Cognitive psychology: A skills approach. Monterey, CA: Brooks/Cole.

Woodworth, R. S. (1938). Experimental psychology. New York: Holt.

Woodworth, R. S. (1940). Psychology. New York: Holt.

Yaniv, I., \& Meyer, D. E. (1987). Activation and metacognition of inaccessible stored information: Potential bases for incubation effects in problem solving. Journal of Experimental Psychology: Learning, Memory, and Cognition, 13, 187-205.

Yarmey, A. D. (1973). I recognize your face but I can't remember your name: Further evidence on the tip-of-the-tongue phenomenon. Memory \& Cognition, 1, 287-290.

Zwicky, A. M. (1982). Classical malapropisms and the creation of a mental lexicon. In L. K. Obler \& L. Menn (Eds.), Exceptional language and linguistics (pp. 115-132). San Diego, CA: Academic Press.

Received August 21, 1989

Revision received April 12, 1990

Accepted April 23, 1990 